\title{
The repulsion effect in preferential choice and its relation to perceptual choice
}

\author{
Mikhail S. Spektor ${ }^{1,2}$, David Kellen ${ }^{3}$, and Karl Christoph Klauer ${ }^{4}$ \\ ${ }^{1}$ Universitat Pompeu Fabra \\ ${ }^{2}$ Barcelona School of Economics \\ ${ }^{3}$ Syracuse University \\ ${ }^{4}$ University of Freiburg
}

Main text: 6945 words

\section{Author Note}

Mikhail S. Spektor, Department of Economics and Business, Universitat Pompeu Fabra and Barcelona School of Economics, Barcelona, Spain. David Kellen, Department of Psychology, Syracuse University, Syracuse, NY, USA. Karl Christoph Klauer, Institute for Psychology, University of Freiburg, Freiburg, Germany.

The main hypotheses, experimental methods, and analysis procedures were pre-registered on the Open Science Framework (available at https://osf.io/4nf2b). Individual behavioral data, pre-processed eye-tracking data, and data-analysis scripts can be found at https://osf.io/9pz58/.

Correspondence: Mikhail S. Spektor, mikhail@spektor.ch 


\begin{abstract}
People rely on the choice context to guide their decisions, violating fundamental principles of rational choice theory and exhibiting phenomena called context effects. Recent research has uncovered that dominance relationships can both increase or decrease the choice share of the dominating option, marking the two ends of an attraction-repulsion continuum. However, empirical links between the two opposing effects are scarce and theoretical accounts are missing altogether. The present study $(N=55)$ used eye tracking alongside a within-subject design that contrasts a perceptual task and a preferential-choice analog in order to bridge this gap and uncover the underlying information-search processes. Although individuals differed in their perceptual and preferential choices, they generally engaged in alternative-wise comparisons and a repulsion effect was present in both conditions that became weaker the more predominant the attribute-wise comparisons were. Altogether, our study corroborates the notion that repulsion effects are a robust and general phenomenon that theoretical accounts need to take seriously.
\end{abstract}

Keywords: repulsion effect, attraction effect, decision making, eye tracking 
Imagine a decision between an apple and an orange. In spite of the famous idiom stating that these two are not comparable, many people face decisions such as this one on a daily basis. These decisions are often influenced by the context in which they take place. For instance, the presence of an additional higher-priced but otherwise equivalent orange - a decoy - can make the original orange appear to be more desirable than before, resulting in a so-called attraction effect (Huber et al., 1982). Figure 1 provides an illustration of the effect. Decades of research have established a plethora of situations under which attraction effects occur, ranging from consumer choice (e.g., Lichters et al., 2017) and risky choice (e.g., Castillo, 2020) to perceptual choice (e.g., Trueblood et al., 2013) and market behavior (Wu \& Yu, 2020), from human decision makers (Gluth et al., 2017) to monkeys (Parrish et al., 2015) and slime mould (Latty \& Beekman, 2011). These findings have quickly led to context effects such as the attraction effect becoming "benchmark" phenomena that any serious theoretical account of multi-alternative decision making needs to successfully capture (e.g., Noguchi \& Stewart, 2018, Soltani et al., 2012; Trueblood et al., 2014, Tsetsos et al., 2012).

At first blush, the attraction effect seems to be a reliable and ubiquitous phenomenon. But a closer look reveals a much more complicated story: On one hand, failed large-scale replication efforts show that the effect is somewhat fragile (Frederick et al., 2014; Yang \& Lynn, 2014). On the other, its manifestation appears to vary wildly across individuals (Liew et al., 2016) as well as being strongly susceptible to changes in the presentation format (e.g., Cataldo \& Cohen, 2019; see Spektor et al., 2021, for a recent overview). In fact, there are circumstances in which one reliably observes a reversed attraction effect - a repulsion effect (Spektor et al., 2018). To date, none of these differences has been directly addressed by the extant theoretical accounts.

An important piece of this puzzle is the fact that the experimental tasks showing reliable attraction effects — vis-à-vis those that do not - happen to differ in a number of ways. For instance, the numerical representation of options' attributes (Frederick et al., 2014, but see Trueblood et al., 2013) or the establishment of indifference between core choice options (Evangelidis et al., 2018; Huber et al., 2014) seem to play critical 


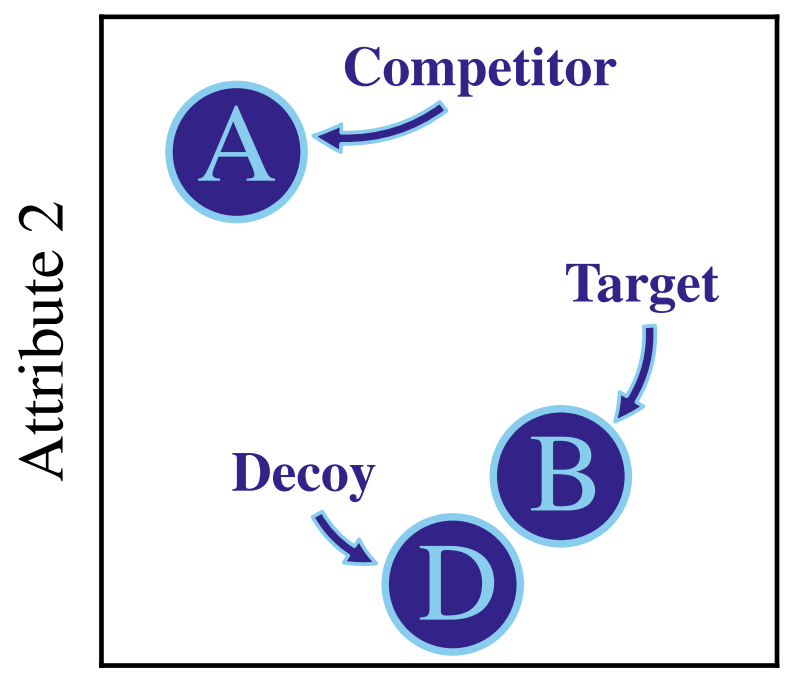

Attribute 1

\section{Figure 1}

Typical attraction-effect setup. All options are characterized by two attributes and there is a trade-off between them. In this example, the decoy option D increases the choice probability of option $B$, the target, at the expense of option A, the competitor.

roles in the observation of attraction effects. Also, some of the weaker attraction effects reported in the literature (Farmer et al., 2017; Trueblood et al., 2013), as well as of the observation of "reversals" such as repulsion effects have mostly been shown in the context of perceptual decision tasks (Evans et al., 2021; Spektor et al., 2018; Trueblood \& Pettibone, 2017). Adding on to these differences are the multiple studies showing stark contrasts between preferential and perceptual choices (e.g., Dutilh \& Rieskamp, 2016; Smith \& Krajbich, 2018, Vanunu et al., 2020; Zeigenfuse et al., 2014). Most noteworthy is a recent study by Liao et al. (2021) that investigated the continuum between attraction and repulsion effects across perceptual, inferential, and consumer-choice tasks. The main take-away of their study is that, while it is possible to observe attraction and repulsion effects within the same task, they do not tend to occur in comparable choice-set compositions, highlighting the lack of generalizability across tasks.

The differences observed between preferential and perceptual decision-making have far-reaching implications for the contemporary study of context effects, given that 
much of its current thrust has been predicated on their interchangeability (e.g., Trueblood et al., 2013; for an overview, see Oppenheimer \& Kelso, 2015). The received view here is normative: Even though preferential and perceptual tasks have different goals, choosing one's preferred option versus choosing the objectively correct option, they nevertheless can be represented as instances of the same optimization problem choosing the option that maximizes some criterion variable. Therefore, they are expected to yield the very same phenomena; in other words, the same context effects. However, this equivalence hinges on abstracted and idealized representations of the experimental tasks (Kellen, 2019), which the above-stated results show to be adrift.

As it stands, there is a clear need for revising contemporary research programs on context effects: At a theoretical level, we can no longer assume that an account motivated by data from a specific domain necessarily applies to another, as if they are somehow tapping into the same set of fundamental, domain-general principles of decision making - they will have to be justified by direct comparisons. At an empirical level, there is a clear need for experimental work that more thoroughly contrasts preferential and perceptual decision making, bringing to light any process-level phenomena (e.g., eye movements; see Noguchi \& Stewart, 2018) that underlie the gaps between them.

The goal of the present work is to contribute on both of these fronts by investigating the attraction-repulsion continuum in a perceptual and a risky-choice task that relies the same stimuli. In terms of theory testing and development, we directly assess the performance of one of the most prominent theoretical accounts of context effects (Trueblood et al., 2014) in its ability to provide an accurate account of behavior in the two different tasks. In terms of empirical results, we provide a characterization of choice behavior and a link to information-processing strategies using eye-movement patterns. Foreshadowing the outcomes of this pre-registered study, we observed repulsion effects in preferential choices, a result that highlights the importance of perceptual features in their manifestation. Furthermore, we found no evidence for qualitative nor substantial quantitative differences in the eye-movement patterns 
observed in the two choice domains, which suggests that individuals tackle both kinds of decisions in a similar fashion. Our model analyses show that the multi-attribute linear ballistic accumulator model (Trueblood et al., 2014), which is typically used to characterize context effects in the type of tasks discussed here, fails to provide an adequate characterization of the data. More specifically, the model is unable to describe the choices - their relative frequencies and associated latencies — that underlie the observed repulsion effects.

\section{Method}

The main hypotheses, experimental methods, and analysis procedures were pre-registered on the Open Science Framework (available at https://osf.io/4nf2b). The present research used only procedures that are exempt from formal ethical approval under the ethical guidelines of the Deutsche Gesellschaft für Psychologie [German Psychological Society]. Data collection was done at the Department of Psychology of the University of Freiburg. Individual behavioral data, pre-processed eye-tracking data, and data-analysis scripts can be found at https://osf.io/9pz58/.

\section{Participants and procedure}

A total of 55 individuals (36 female, 19 male, age 18-35, $M=23.80$, $S D=3.85$ ), mainly students of psychology from the University of Freiburg, were included in the final sample size (see exclusion criteria below). The study consisted of two sessions that were separated by at least one week from each other. After giving informed consent, participants were seated in a single-person lab room in front of a $47.38 \times 29.61 \mathrm{~cm}$ screen $(22$ " screen diagonal) with a resolution of $1680 \times 1050$ pixels. Each session began with a demographic questionnaire (age, sex, subject of study or profession, handedness, and presence of color blindness) followed by instructions and an example trial. At the beginning of the experimental phase, the first eye-tracking calibration and validation of said calibration took place.

Each trial began with a fixation cross displayed at the center of the screen. Individuals' gazes had to continuously stay within a circular area of interest (AOI) with 


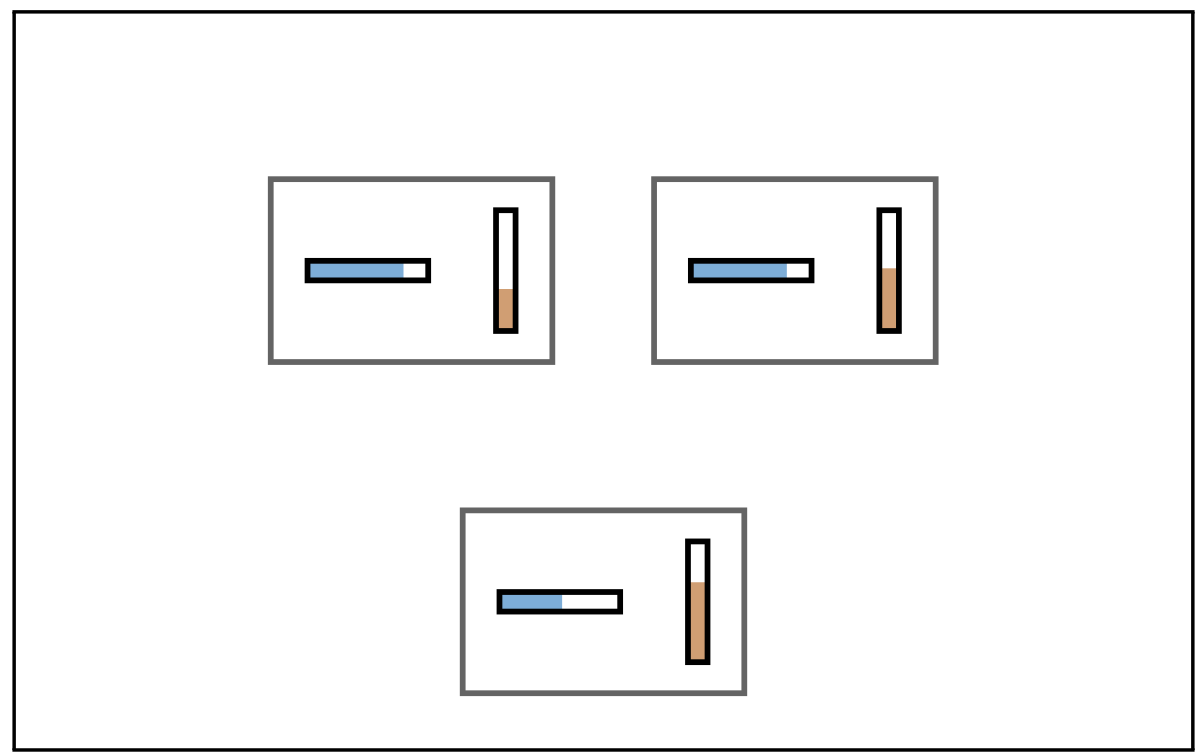

Figure 2

Example of a choice trial. Depicted is the arrangement in a downward-facing triangle (counter-balanced across participants and sessions).

a radius of 100 pixels for $500 \mathrm{~ms}$. The eye tracker was recalibrated if that criterion was not reached within 12 s. After the fixation cross disappeared, three options with two attributes each appeared at the edges of an equilateral triangle around the center of the screen. The attributes were represented by differently colored, partially filled horizontal and vertical bars (see Figure 2 for an example of a trial). Individuals had up to $5 \mathrm{~s}$ to make a choice. If a choice has not been made within this time, individuals saw a screen stating that they were too slow and should respond faster. After every trial, a blank screen was shown for another 750-1,250 ms and a new trial began. Each of the two experimental sessions contained 700 trials and every 175 trials, individuals had the opportunity to make self-timed breaks. After every break, the eye tracker was recalibrated. Sessions were on average 67 minutes long ( $S D=11$ minutes). At the end of session 2, individuals obtained a show-up fee of the course-credit equivalent of three hours or $21 €$ and a bonus payment ranging from $0 €$ to $4 €$. 


\section{Experimental task and design}

The experimental task was either a risky choice between three lotteries with two equiprobable outcomes each or a perceptual length-discrimination task. In case of a risky-choice task, the levels of the horizontal and vertical bars coded outcome magnitudes $x$ and $y$, respectively. ${ }^{1}$ A full bar corresponds to $2 €$, whereas an empty bar corresponds to $0 €$. Such bar-based representations are quite common in the risky-choice literature (e.g., Farmer et al., 2017; Gluth et al., 2018; Spitmaan et al., 2019; Tsetsos et al., 2016). In the case of a perceptual framing, individuals were instructed to choose the option whose bars were filled the most (i.e., with the highest sum). For incentive-compatibility, a correct choice corresponded to $2 €$, a choice of the option with the second-highest sum corresponded to $1 €$, and $0 €$ otherwise, in case the trial was selected for the bonus payment. Note that an optimal agent will choose the same options in both conditions.

The experimental design closely followed that of Spektor et al. (2018), with a total of seven within-subject factors:

i) Condition: whether the task was framed as a perceptual or preferential.

ii) Correct Option: which of the two core options had the larger criterion value (i.e., sum of their two attributes). The wide-but-low (WL) option whose horizontal bar was filled more than its vertical bar or the narrow-but-high $(\mathrm{NH})$ option whose vertical bar was filled more than its horizontal bar.

\footnotetext{
1 Traditionally, context-effect research in risky choice has relied on choices between lotteries that are characterized by a single non-zero outcome and its associated probability (e.g., Farmer et al., 2017, Herne, 1999. Izakson et al., 2020; Soltani et al., 2012 Tversky, 1972). The idea underlying these studies is that people treat the two pieces of information that they see (i.e., the outcome of a lottery and its probability of occurrence) as separate attributes, much like they would treat the price and quality of a consumer-choice article as two separate attributes. Instead of a non-zero outcome and its probability, the present study varies the possible outcomes of a 50-50 lottery, an approach that is very commonly used in the risky-choice literature (e.g., Kellen et al., 2020; Tom et al., 2007; Tsetsos et al., 2012; Tsetsos et al., 2016; Walasek \& Stewart, 2015).
} 
iii) Set Type: whether the inferior of the two core options was inferior on the horizontal or vertical attribute.

iv) Difficulty: whether the two options differ by $3 \%$ or $7 \%$ of the maximum theoretical criterion value of 400 .

v) Target Option: whether the decoy option is similar-but-inferior to WL or NH (i.e., which option was made the target due to the presence of the decoy; the other option is called the competitor).

vi) Decoy Type: whether the decoy was inferior on the target's stronger attribute (frequency decoy), the target's weaker attribute (range decoy), or both of the attributes (range-frequency decoy).

vii) Attribute Distance: whether the decoy differed from the target by $2 \%, 5 \%$, or $9 \%$ of the maximum theoretical criterion value of 400 .

For each of the eight Correct Option $\times$ Set Type $\times$ Difficulty factor combinations, we created four sets of core options. We did so by drawing 32 independent values from uniform distributions with ranges $[160,200]$ and $[70,110]$ to create the WL options. The NH options consisted of WL options with reversed attribute values. To each of the options, we applied one of the eight factor combinations to the respective core option. To each of the resulting unique core sets, we then applied all of the remaining 18 factor combinations, resulting in 576 unique experimental trials. Additionally, we created another 124 so-called catch trials that were particularly easy (Difficulty $=15 \%$, Attribute Distance $=20 \%$ ) and were used to assess accuracy for excluding participants. The same set of stimuli was used in both conditions and the order of presentation was randomized for each session.

We counterbalanced across participants the condition with which they started, the associations of colors to the horizontal and vertical bars, the locations of the horizontal and vertical bars (i.e., whether the horizontal bar was to the left or the right of the option's center), and the arrangement of stimuli on-screen (i.e., whether they 
were arranged in a downward-facing triangle as depicted in Figure 2 or an upward-facing triangle). All of these balancing factors were reversed in the second session: For instance, an individual who started with the preferential condition with the horizontal bar on the left, the vertical bar being orange, and a downward-facing triangular arrangement, later completed the perceptual condition with the horizontal bar being orange and on the right, and the options arranged in a upward-facing triangle.

Finally, eye-movement data was recorded using an SMI RED500 eye-tracking device. As a calibration algorithm, we used the built-in five-point algorithm. All measurements were initially recorded using a sampling rate of $500 \mathrm{~Hz}$. In case of tracking problems or frequent recalibrations, we lowered the sampling rates to $250 \mathrm{~Hz}$, $120 \mathrm{~Hz}$, and finally $60 \mathrm{~Hz}$, in that order. Raw gaze positions were re-coded into events (fixations, saccades, and blinks) using SMI's BeGaze3 software package and the high-speed detection algorithm (whenever possible, otherwise the low-speed algorithm) and default values. We defined three AOIs for each of the options. The first AOI reflected the option as whole and was identical to the frame surrounding the two attributes. The second AOI covered all of the horizontal bar's area and additional 20 pixels around it. The third AOI covered all of the vertical bar's area and additional 20 pixels around it. The first AOI includes the other two that were not overlapping. All fixations outside of the pre-defined AOIs were counted as empty gazes.

\section{Exclusion criteria}

A total of 97 individuals registered and showed up for the first session of the experiment. As per pre-registration, we excluded participants based on their performance in the catch trials. Sixteen participants who did not choose the clearly best option (i.e., the option with the highest expected value in the preferential-choice task or the highest sum of attributes in the perceptual task) in at least two thirds of the cases were excluded from all analyses. Due to technical problems with the eye-tracking hardware, an additional 26 participants were excluded who did not complete both experimental sessions (14 of which could complete the first but not the second session 
and 12 of which could not complete the first session), resulting in a final sample size of 55 individuals.

In addition to excluding participants based on the above-mentioned criteria, we also excluded single trials from the participants retained for analysis. Following Spektor et al. (2018), we excluded $753(0.98 \%)$ trials in which participants responded too fast (i.e., faster than $100 \mathrm{~ms}$ ). Due to the deadline of 5,000 ms in the experimental task, another $735(0.95 \%)$ trials were excluded. Aside from these exclusions, all other trials were included in our analyses of choice behavior. For the analyses that involved eye-movement measures, we excluded all trials for which the dependent variable did not have a single valid observation. For example, when we investigated transitions between attributes or options, we did not include trials in which there were no transitions between these AOIs.

\section{Choice behavior}

As our core behavioral measure, we relied on the relative choice share of the target (RST), a standard measure of context effects that controls for prior biases (perceptual condition) or risk attitudes (preferential condition):

$$
\mathrm{RST}=\frac{\operatorname{Pr}(\text { target })}{\operatorname{Pr}(\text { target or competitor })},
$$

where $\operatorname{Pr}$ denotes the probability of choosing a specific option. The main behavioral analyses were related to whether individuals show context effects in the two conditions. RST values are defined on the unit scale, and an absence of context effects is reflected in an $\mathrm{RST}$ value of $\frac{1}{2}$.

The RST values were modeled under a Bayesian framework, using a probit link function that allowed the proportions on the $[0,1]$ scale to be characterized on a real scale. Specifically, we fitted a bivariate Normal distribution with a vector of means $\boldsymbol{\mu}$, one mean for the preferential-choice condition and one for the perceptual-choice condition, and variance-covariance matrix $\Sigma 2^{2}$ The main parameters of interest are $\boldsymbol{\mu}$. If the $95 \%$ BCI of the elements of $\boldsymbol{\mu}$ overlap with 0 on the real line (corresponding to an

\footnotetext{
${ }^{2}$ As priors, we used independent standard normal distributions for $\boldsymbol{\mu}$. In the case of $\Sigma$, we decomposed
} 
overlap with .5 on the unit scale), then there is no context effect. If it is above 0 on the real line, then there is an attraction effect (i.e., RST $>\frac{1}{2}$ ). If it is below 0 on the real

line, then there is a repulsion effect (i.e., reversed attraction effect; RST $<\frac{1}{2}$ ). The rank stability of the RSTs between individuals and conditions was captured by the covariance term in $\Sigma_{r}$. Finally, we investigated how attraction/repulsion effects evolved across conditions by means of a 2 (Condition) $\times 3$ (Attribute Distance) within-subject factorial decomposition of $\Phi^{-1}$-transformed RST values.

\section{Joint modeling of choices and latencies}

In order to obtain a more principled characterization of individuals' choice behavior and their associated latencies, we fitted the multiattribute linear ballistic accumulator model (MLBA; Trueblood et al., 2014). The MLBA is an extension of the linear ballistic accumulator model (LBA; Brown \& Heathcote, 2008), a model from the class of evidence-accumulation models, according to which decision makers accumulate noisy pieces of evidence until a predetermined decision criterion is reached and the response is executed. The LBA assumes separate accumulators for each available choice alternative. These accumulators start accumulating evidence from a starting point between 0 and $A$ with a constant accumulation rate $\eta$, the drift rate. As soon as the threshold $k$ is reached, a response is initiated. Response execution and other non-decision related processes are captured in the $\tau$ parameter.

The MLBA provides a front-end to the LBA according to which the options' attributes interact with each other such that the drift rate $\eta$ is context-dependent. Roughly, this process works as follows (see Trueblood et al., 2014, for details): Objective inputs are transformed to subjective perceptions using a psychophysical function that

$\Sigma$ into a correlation matrix $\Sigma_{r}$ and a scaling vector $\zeta$. For the correlation matrix $\Sigma_{r}$, we used the uniform LKJ(1) prior. For the scaling vector $\boldsymbol{\zeta}$, we used two independent half-normal distributions with standard deviations of 1.5. We used the No-U-Turn sampler as implemented in Stan (Carpenter et al., 2017) via the PyStan interface for Python to obtain samples from the posterior distributions. Inferences were based on a comparison between the 95\% Bayesian credible intervals (BCI) of the posterior distributions. Frequentist tests led to qualitatively identical conclusions and will not be reported here. 
includes an extremeness aversion parameter $m$. Options' attribute perceptions are compared with each other and receive a weight that exponentially declines according to a parameter $\lambda$. The MLBA assumes that winning comparisons and losing comparisons are weighted differently (via parameters $\lambda^{+}$and $\lambda^{-}$). The possibility that individuals prioritize one attribute over the other is captured using a bias parameter $\beta$. Finally, a scaling parameter $I_{0}$ is added to each options' valuation to form the drift rate. Additionally, we estimated the recently proposed $\gamma$ parameter which scales the drift rates and has been shown to improve model fits (e.g., Evans et al., 2019). In sum, the MLBA establishes parameters $m, \lambda^{+}, \lambda^{-}, I_{0}, \beta$, and $\gamma$ at the front-end and $k, A$, and $\tau$ at the LBA back-end. For the back-end component, we modified the LBA code provided in Annis et al. (2017). At the front-end, we linearly scaled the options' attributes down to the $[0,10]$ range, as recommended by Trueblood (2015, personal communication). Unless stated otherwise, catch trials were excluded from the model fitting.

\section{Transition patterns}

In line with past research (Noguchi \& Stewart, 2014, 2018), we investigated the comparison processes underlying multi-alternative, multi-attribute choice using profiles of gaze transitions. To do so, we defined four different transition types, which are illustrated in Figure 3. The first two are transition patterns that correspond to a single transition:

1. Attribute-wise transitions that correspond to transitions across two options within the same attribute,

2. Alternative-wise transitions that correspond to transitions across the two attributes within an option,

The other two further specify behavior following attribute-wise transitions; Do individuals follow up with another transition to the same attribute of the third option or do they switch to a different attribute?

3. Pure attribute-wise transitions that correspond to transitions across all three 
options within the same attribute (note that pure attribute-wise transitions comprise of two specific attribute-wise transitions), and

4. Attribute-and-alternative-wise transitions that correspond to pairs of attribute-wise transitions on different attributes.

We used hierarchical binomial models to investigate which transition patterns are more frequent and the relative stability of the transition patterns across individuals and conditions. The first analysis involved attribute-wise and alternative-wise transitions. The second analysis investigated the transitions following attribute-wise transitions more in detail by contrasting pure attribute-wise transitions with attribute-and-alternative-wise transitions. The third analysis broke down attribute-and-alternative-wise transitions into within-alternative and across-alternative transitions following the attribute-wise transition.

In order to relate the different eye-movement patterns to individuals' choices, we used a hierarchical logistic regression within each condition separately. In this model, the probability of choosing the target option (vs. the competitor) is predicted using an intercept and the following predictors: (a) difference in the relative dwelling times between the target and the competitor, (b) absolute number of transitions within options, (c) absolute number of transitions within attributes, and (d) whether the last fixated option was the target or not. The latter predictor was included to control for the gaze-cascade effect (Shimojo et al., 2003), according to which individuals are likely to choose the option they fixate last (although the direction of the causality is controversial to date). All predictors were averaged across the recordings of both eyes and scaled afterward. Missing values (only present in the last predictor whenever there were no valid fixations recorded in a trial) were replaced by 0 such that the variable had no influence on the respective trials. We used independent normal distributions as group-level distributions with $\mathcal{N}(0,2)$ priors on the means and corresponding half-normal distributions for the standard deviations. 

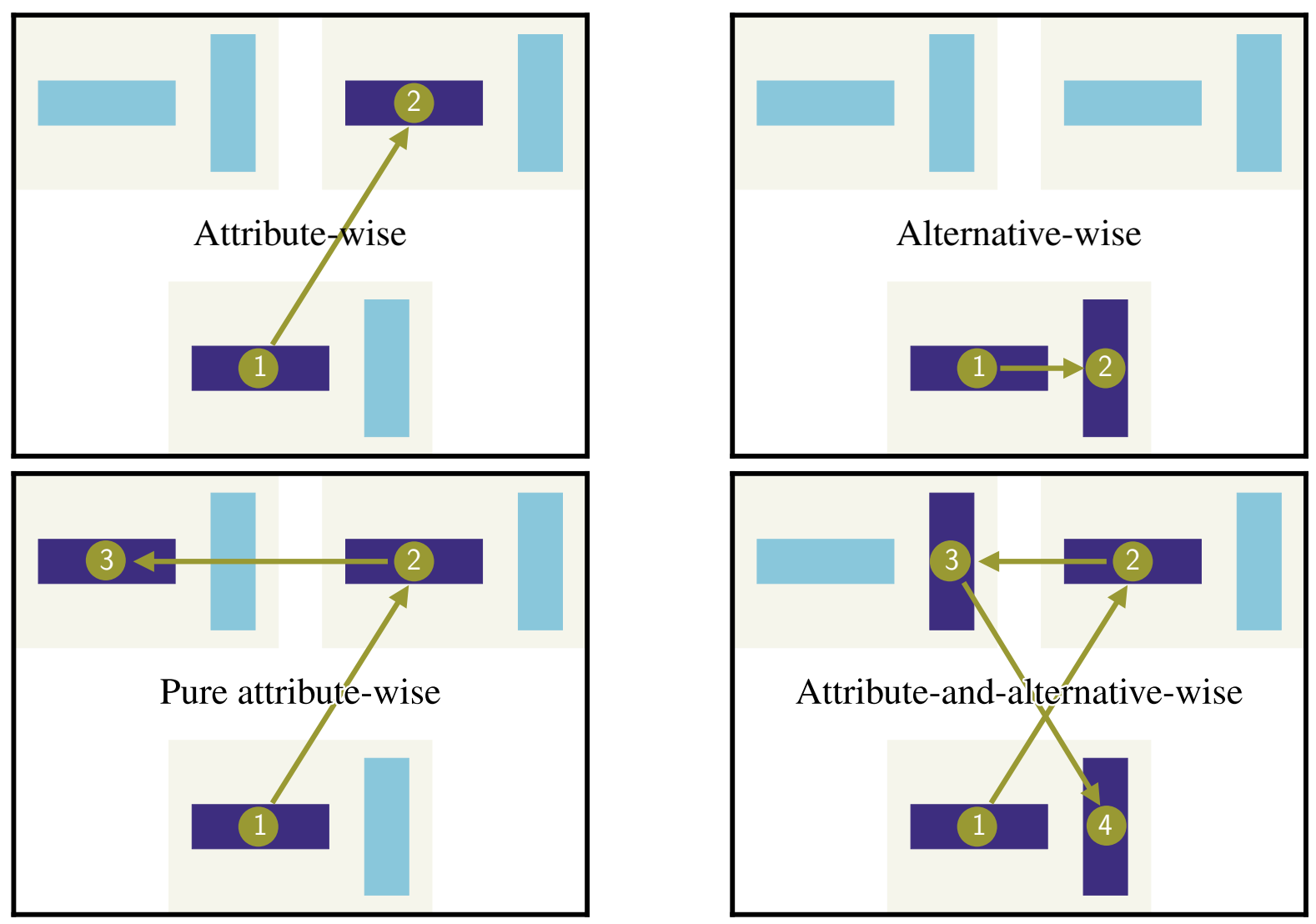

\section{Figure 3}

Different transition types considered in the analyses. Numbers indicate the order of fixations and arrows indicate transition directions. Depicted is the situation in which the options are arranged in a downward-facing triangle.

\section{Results}

\section{Descriptive statistics}

At a descriptive level, we found a classical speed-accuracy trade-off in favor of faster decisions (and lower accuracy) in the preferential condition: In the perceptual condition, they chose the option with the highest criterion value in $69.5 \%$ of the trials, the option with the second-highest criterion value in $19.8 \%$ of the trials, the lowest-criterion option in $9.3 \%$ of the trials, and responded too slowly in $1.5 \%$ of the trials. They were slightly less accurate in the preferential condition, with choice proportions of $65.8 \%, 22.5 \%, 11.2 \%$, and $0.8 \%$, in the same order. This decrease in 
accuracy was accompanied by a decrease in response times, where an average trial took $2398 \mathrm{~ms}$ in the perceptual condition and only $2082 \mathrm{~ms}$ in the preferential condition. Additionally, individuals exhibited a somewhat low degree of choice consistency (i.e., the proportion of trials on which individuals chose the same option given the same stimuli across the two conditions), averaging at $55 \%$ and with a sizeable degree of individual differences $(S D=10 \%) .^{3}$ In sum, individuals performed the task quite well and behavior between the conditions differed in purely descriptive aspects, suggesting that the participants did not apply the same decision criterion across conditions but rather treated preferential and perceptual decisions distinctly.

\section{Repulsion effects in both conditions}

As our main hypothesis, we expected context effects to arise in both the preferential-choice and the perceptual conditions. Based on the vast literature on the attraction effect in preferential choice, we expected the attraction effect to arise in the preferential-choice condition. Based on an exploration of the attraction-repulsion continuum in a different perceptual decision-making task (Spektor et al., 2018), we expected the repulsion effect to occur in the perceptual condition.

In contrast to our expectations, we observed the repulsion effect in both conditions. In the perceptual condition, the median of the RST was .481 (95\% BCI: $[.474, .487])$, corresponding to a medium-to-large effect size of $d=0.773$ (95\% CI: [0.468, 1.071]). In the preferential condition, the median RST was .487 (95\% BCI: [.475, .498]), corresponding to a small-to-medium effect size of $d=0.320$ (95\% CI: $[0.048,0.590])$. The RSTs in the preferential condition were on average higher, $M d n=.006(95 \%$ BCI: $[-.004, .017])$, although the $95 \%$ BCI overlapped with 0 . This was reflected in a small effect size for which the CI overlapped with $0, d=0.169$ (95\% CI: [-0.097, 0.435]).

\footnotetext{
${ }^{3}$ Although this $55 \%$ average is substantially higher than the expected consistency under random responding of $33.3 \%$, it is low in relation to the $63 \%(S D=10 \%)$ and $60 \%(S D=11 \%)$ best-choice response probabilities observed in the perceptual and preferential conditions, respectively, when only considering the most difficult trials (in which choice variability is expected to be the highest; see
} Figure 4). 
Across the conditions, RST values were relatively stable, with a median correlation of $M d n_{r}=.443\left(95 \% \mathrm{BCI}_{r}:[.210, .638]\right)$. Results do not change qualitatively when analyzing the first session only, alleviating the concern that the repulsion observed across conditions is due to order or transfer effects (see Appendix for details).

The repulsion effect is present across all levels of the attribute-distance factor in both conditions (see Figure 4a); However, it is weakest when the decoy is $9 \%$ worse than the target $(\mathrm{RST}=.496$ and $\mathrm{RST}=.495$ in the perceptual and preferential condition, respectively) and strongest in the factor level where the target and the decoy differ by only $2 \%(\mathrm{RST}=.461$ and $\mathrm{RST}=.478$ in the perceptual and preferential condition, respectively). This observation is in line with previous findings (Spektor et al., 2018) and is supported by a Bayesian 2 (Condition) $\times 3$ (Attribute Distance) within-subject ANOVA on the $\Phi^{-1}$-transformed RST values, where the best model is one that includes a main effect of attribute distance, $\mathrm{BF}_{10}=4423 \pm 1.27 \%$ (compared to the random intercept-only model). A similar analysis involving the type of the decoy (i.e., range decoy, frequency decoy, or range-frequency decoy) showed no clear effect of decoy type, in which a random intercept-only model was better than all other models, $\mathrm{BF}_{10}=0.726 \pm 0.77 \%$ (compared to the model with a main effect of decoy type). We report additional analyses in the Appendix section 'Characterizing the repulsion effect]'.

\section{The multiattribute linear ballistic accumulator model provides a poor account of the data}

We attempted to obtain a model-based characterization of individuals' behavior in terms of latent cognitive processes. To do so, we relied on the MLBA model (Trueblood et al., 2014), a model that has been frequently used to model multi-attribute, multi-alternative choices in both the preferential (Cataldo \& Cohen, 2020; Turner et al., 2018) and the perceptual domain (Evans et al., 2019; Spektor et al., 2018). Usually, the MLBA has been shown to outperform its competitors when characterizing choices as well as their associated latencies (Cataldo \& Cohen, 2020; Evans et al., 2019, but see Cohen et al., 2017; Turner et al., 2018, for cases where it did 
(a)

Perceptual condition
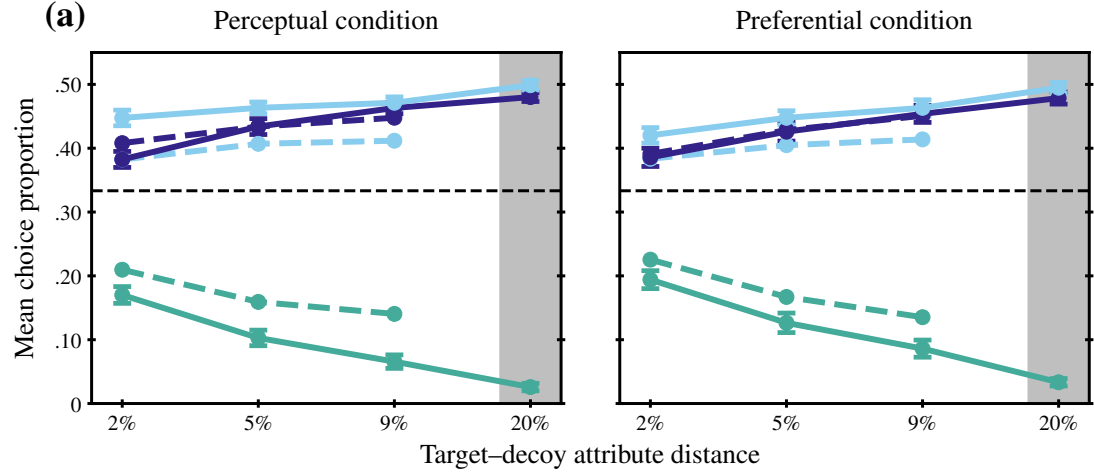

Target

Competitor

Decoy

- Data

- MLBA predictions

(b)
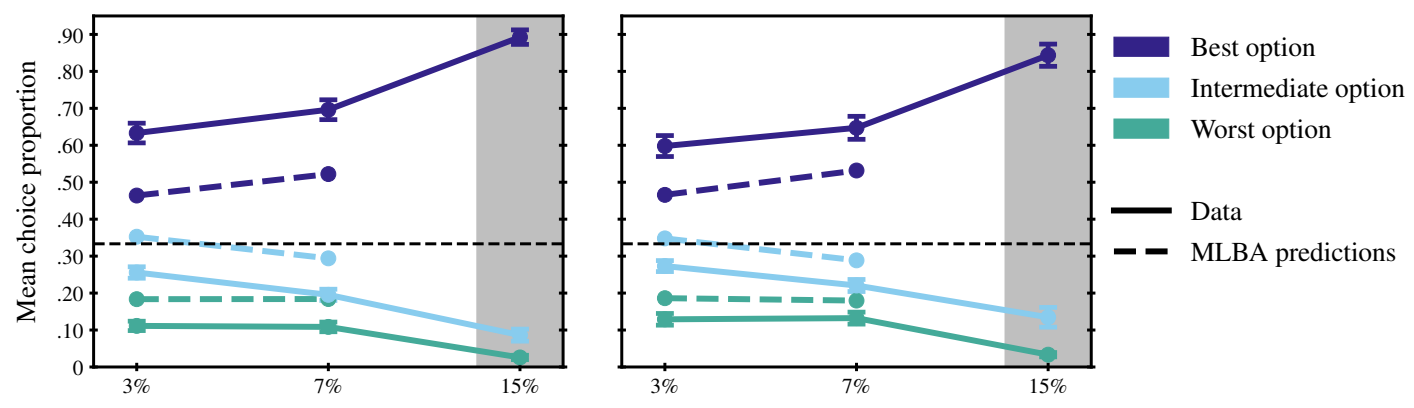

Difficulty

\section{Figure 4}

Mean choice proportions of the options and corresponding model predictions, split by condition and (a) target-decoy attribute distance and (b) choice difficulty. Target is the option that is similar to the decoy option but dominating it, whereas competitor is the option that is dissimilar to both target and decoy. All distance measures are in percentages of the maximum criterion value of 400. The conditions that were used as catch trials are highlighted by a shaded background and were excluded from model fitting. Model predictions reflect mean posterior predictions and are represented by the dashed lines. Error bars indicate the $95 \%$ CI of the mean.

not perform that well).

We fitted the full MLBA model to the individual level for each condition separately (see Method section for details). We used the joint posterior distribution to draw inferences about the model's capability to account for the data. At first, we looked at the concordance of the predicted choices with actual choices of the participants. The predictive accuracy of choices was somewhat poor for a majority of participants, ranging from $36 \%$ to a maximum of $62 \%$ (averaging at $45 \%, S D=8 \%$ ), compared to 
the chance level of $\frac{1}{3}$. A closer look at the model predictions reveals that the MLBA not only fails at a quantitative level but also qualitatively: The model is unable to predict the repulsion effect at all and cannot capture the effects of attribute distance (Figure 4 a, dashed line) or difficulty (Figure 4b, dashed line) on choices, with almost all predictions falling outside the $95 \%$ CI of the observed choice proportions. These failures also extended to the choice latencies, as shown in Figure 5 where we plot the mean predicted response times of the MLBA (irrespective of the choice) against the empirical response times. Due to these shortcomings of the standard implementation, we refrain from discussing the MLBA's latent characterization of the data using the estimated parameter values. 4

To explore the reasons underlying the difficulties of the standard implementation of the MLBA model, we pursued two strategies: 1) fitting more flexible MLBA specifications that can account for larger varieties of data patterns, and 2) simplifying the prediction problem, so that MLBA only has to predict discrete choices, leaving out their associated response latencies. Both strategies led to model-performance improvements, but at the cost of a seven-fold increase of the number of free parameters (Figure A4) or the ability to jointly account for choices and their latencies (Figure A6). Importantly, the more flexible MLBA still struggled with some behavioral patterns, whereas the MLBA that only fitted discrete choices provided an excellent fit to the data. These differences enable us to trace back the MLBA's shortcomings to its joint account of discrete choices and associated latencies. Details are provided in the Appendix section "Alternative specifications of the multiattribute linear ballistic accumulator modell'.

\footnotetext{
${ }^{4}$ We have no reasons to believe that this failure is due to the implementation of eye-tracking (for a successful application, see Gluth et al., 2018). Moreover, a close inspection of the response times did not reveal any anomalous patterns.
} 


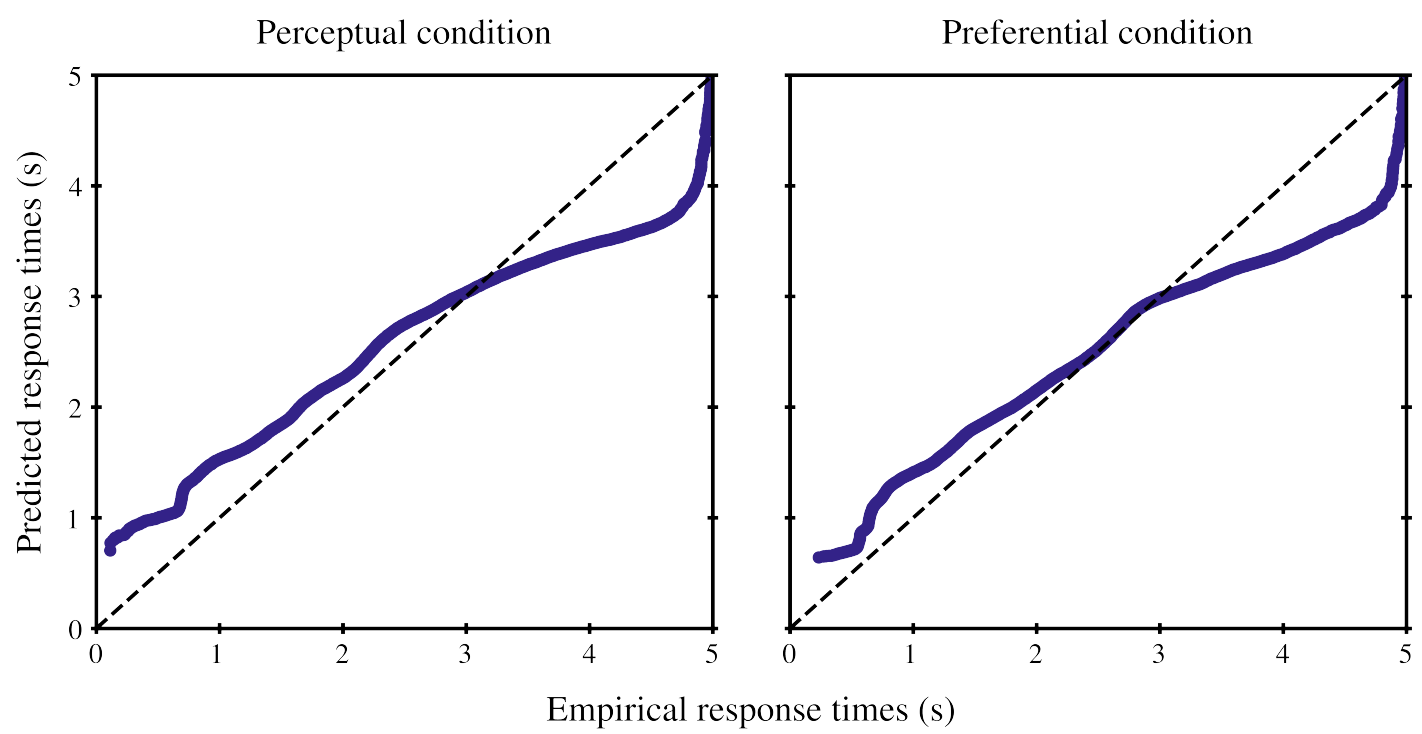

\section{Figure 5}

Quantile-quantile plots of empirical and predicted response times. Predictions are based on the mean of the posterior response-time predictions of the multiattribute linear ballistic accumulator model.

\section{Individuals engage mostly in alternative-wise comparisons}

One of the main goals of the present study was to relate individuals' tendencies to make context-dependent choices with their information-search strategies and assess their stability across the conditions. To do so, we recorded participants' eye movements using a high-speed eye-tracking device during both experimental sessions. We relied on a transition-based classification (Noguchi \& Stewart, 2014) to classify the information-search strategies using a total of four different transition types (see also the Method section and Figure 3.

The most general distinction can be made by relying on transition patterns that involve only a single transition (based on an average of 443 trials with at least one of these two transition types per individual and condition). We evaluated whether individuals are more likely to engage in attribute-wise comparisons (transitions across two options within the same attribute) or alternative-wise comparisons (transitions across the two attributes within an option). We used a hierarchical Bayesian binomial model to assess, in each condition, which of the two transitions is more frequent and, 
between conditions, whether there is a rank stability across individuals. In other words, does an an individual that engages in attribute-wise transitions more often than other individuals in one condition also engage more often in attribute-wise transitions in the other condition?

We found that individuals were more likely to perform within-alternative than within-attribute transitions; The group-level probability of a within-alternative transition was above $50 \%$ in both the perceptual $(M d n=.576,95 \%$ BCI: $[.550, .601])$ and preferential conditions $(M d n=.54695 \%$ BCI: $[.514, .576])$. However, individuals were less likely to perform within-alternative transitions in the preferential condition, with $M d n=-.030,95 \%$ BCI: [-.057, -.004]. Finally, individuals showed a substantial degree of rank stability across the conditions, with $M d n_{r}=.606,95 \% \mathrm{BCI}_{r}$ : [.388 .760].

In a next step, we assessed which kind transition follows a within-attribute transition, either a transition to the third option on the same attribute (pure attribute-wise transition) or a transition to the different attribute (of any option; based on an average of 113 trials with at least one of these two transition types per individual and condition). We found that pure attribute-wise transitions were less frequent than attribute-and-alternative-wise transitions in both conditions, where the probability was slightly higher $(M d n=.031,95 \%$ BCI: $[.006, .058])$ in the preferential condition $(M d n=.375,95 \%$ BCI: $[.379, .434])$ than in the perceptual condition $(M d n=.375$, 95\% BCI: $[.348, .402])$. Rank stability across individuals was very high $\left(M d n_{r}=.830\right.$, $\left.95 \% \mathrm{BCI}_{r}:[.528, .973]\right)$.

In a final step, we checked which transition was most common in such attribute-and-alternative-wise comparisons: A transition to the other attribute on the same option or a different option (based on an average of 80 trials with at least one of these two transition types per individual and condition). In this case, almost all transitions following a within-attribute transition in the perceptual condition $(M d n=.828,95 \%$ BCI: $[.805, .850])$ and in the preferential condition $(M d n=.812$, $95 \%$ BCI: $[.788, .834])$ went to the other attribute of the same option, a tendency that did not differ between conditions $(M d n=-.016,95 \%$ BCI: [-.039, .007]). Much like 
with the other analyses, rank stability was very high across individuals, with $M d n_{r}=.864,95 \% \mathrm{BCI}_{r}:[.485, .987]$.

Altogether, individuals were most likely to integrate the two attributes within an option before transitioning to a different option and integrating its attributes within the option. These tendencies demonstrated a substantial rank stability, such that individuals who were more likely to perform each of the analyzed patterns in one condition were also those who were more likely to do the same in the other condition. However, there were two main effects of condition, such that the dominating pattern summarized in this paragraph occurred more frequently in the perceptual condition than in the preferential condition.

\section{Absence of attribute-wise comparisons drives the repulsion effect}

We related the degree to which individuals were sensitive to the choice context to their respective eye-movement patterns. To do so, we ran a hierarchical Bayesian logistic regression in which the choice of the target (vs. the competitor) was predicted by the difference in the relative dwelling times between the target and the competitor, the absolute number of transitions within options, the absolute number of transitions within attributes, and whether the last fixated option was the target or not. The resulting posterior distributions of the group-level means are reported in Table 1.

In line with past research, the longer people looked at the target (compared to the competitor), the more likely they were to choose it (as reflected in the group-level mean that is entirely positive). Additionally, individuals exhibited the gaze-cascade effect (Shimojo et al., 2003), according to which the last fixation is a strong predictor of the ultimate choice. The critical novel observation is the relationship between the number of within-alternative and within-alternative transitions on the propensity of choosing the target option. In both conditions, the number of within-alternative transitions is not related to the probability of target choices, as indicated by a wide credible interval that includes 0 . However, the more frequently individuals compared the same attribute across different options, the more likely they were to choose the 


\begin{tabular}{ccrrrr}
\hline Condition & Predictor & $M$ & $S D$ & $2.5 \%$ & $97.5 \%$ \\
\hline \multirow{4}{*}{ Perceptual } & Intercept & -0.073 & 0.018 & -0.108 & -0.039 \\
& $\Delta_{\text {target, competitor }}$ & 0.808 & 0.096 & 0.614 & 0.989 \\
& $N_{\text {within-alternative }}$ & -0.032 & 0.019 & -0.067 & 0.007 \\
& $N_{\text {within-attribute }}$ & 0.072 & 0.019 & 0.037 & 0.109 \\
& Last fixation on target & 0.656 & 0.052 & 0.550 & 0.760 \\
\hline \multirow{3}{*}{ Preferential } & Intercept & -0.025 & 0.028 & -0.084 & 0.024 \\
& $\Delta_{\text {target, competitor }}$ & 0.934 & 0.109 & 0.745 & 1.168 \\
& $N_{\text {within-alternative }}$ & -0.008 & 0.019 & -0.043 & 0.031 \\
& $N_{\text {within-attribute }}$ & 0.075 & 0.019 & 0.038 & 0.113 \\
& Last fixation on target & 0.731 & 0.056 & 0.636 & 0.851 \\
\hline
\end{tabular}

\section{Table 1}

Posterior distribution of the group-level parameters of the logistic regression.

$\Delta_{\text {target, } \text { competitor }}=$ difference in the relative dwelling times between the target and the competitor. $N_{\text {within-alternative }}=$ absolute number of transitions within options.

$N_{\text {within-attribute }}=$ absolute number of transitions within attributes. Columns 2.5\% and 97.5\% indicate the lower and the upper boundaries of the $95 \%$ highest-density interval, respectively.

target option. In other words, within-attribute transitions weaken the repulsion effect.

\section{Discussion}

The present study reports the first within-subject experimental investigation of the attraction-repulsion continuum that links choice behavior to information-processing strategies using eye-movement patterns. It is also among the first to demonstrate the repulsion effect in a preferential task. The eye-movement patterns suggest that repulsion effects are driven by a lack of attribute-wise comparison processes and that information-search strategies do not differ between the two conditions in a meaningful 
way. Our results indicate that perceptual features of preferential tasks have a non-negligible influence on choices and the necessity for theories that take the presentation format into account.

\section{Eye movements underlying context effects}

The results reported here advance our understanding of context effects in the perceptual domain. Apart from few exceptions (Tsetsos et al., 2012), context-effect research in the perceptual domain was conducted using a task in which individuals had to indicate the largest of three rectangles (e.g., Farmer et al., 2017; Liao et al., 2021;

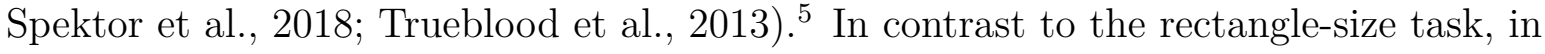
which width and height are visually inseparable attributes, the task introduced here permits tracking the attribute that individuals fixated. The ability to separate fixations between the different attributes allowed for a comparison with previous studies in preferential choice, most notably Noguchi and Stewart (2014). In contrast to the present study, Noguchi and Stewart (2014) found that attribute-wise transitions were more common than option-wise transitions. What seems like a contradiction at first turns out to corroborate our conclusions: We found a positive relation between attribute-wise transitions and the propensity to choose the target option. The imbalance of transitions in favor of option-wise transitions goes in line with the fact that Noguchi and Stewart (2014) observed an attraction effect, whereas we observed a repulsion effect. The remaining eye-tracking results are very similar between their studies and ours, which suggests that (absence of) attribute-wise transitions are the main driving force behind (repulsion) attraction effects (Cataldo \& Cohen, 2019). Note that the manipulation of the presentation format reported by Cataldo and Cohen (2019), and their re-analysis of Noguchi and Stewart's data, is consistent with the present eye-tracking results: In their study, a condition that emphasized alternative-wise comparisons led to weaker

\footnotetext{
${ }^{5}$ It should be noted that Liao et al. (2021) reported an experiment in which individuals had to select the largest among a set of ovals (Exp. 1b), in addition to an experiment with rectangles (Exp. 1a). Just like in the rectangle-size task, the ovals were characterized by an option-specific height and width, so the same line of reasoning applies to that task as well.
} 
attraction effects than a condition that emphasized attribute-wise comparisons. Even though Cataldo and Cohen (2019) did not find a repulsion effect, their results, when taken together with ours, suggest that endogenous and exogenous variation in the comparison process affects behavior similarly.

The way stimuli were displayed on-screen might have facilitated within-alternative transitions as attributes pertaining to any given option were closer to each other than attributes of different options. However, this cannot explain the

difference between our results and previous studies, as Noguchi and Stewart (2014) had a similar arrangement. Even though it would be possible to present the stimuli in a way in which the attributes within an option are equally distant from each other as from a different option (e.g., by placing the stimuli in a horizontal line next to each other), we believe such a format to be less appropriate than the one we used. First, it is a more realistic arrangement that corresponds to most decision-making settings in which there is a larger physical distance between options than between attributes within options. Second, such an arrangement would introduce other biases in eye movements (e.g., transitions between two options next to each other are easier than between the outer options) and choices (Spektor et al., 2018). Altogether, the present results buttress Spektor et al.'s (2018) conclusions about the importance of perceptual task features at the level of eye movements.

\section{The repulsion effect across domains}

Until recently, the repulsion effect was considered a fragile phenomenon (Simonson, 2014) which only occurred as a non-systematic fluctuation around a null effect across studies (Frederick et al., 2014). This notion was challenged by demonstrations of robust repulsion effects using perceptual (Evans et al., 2021; Liao et al., 2021; Spektor et al., 2018), inferential (Liao et al., 2021), and consumer-choice tasks (Liao et al., 2021). The present repulsion effect in a risky-choice task further extends this list and corroborates the empirical status of repulsion effects, prompting the question: What exactly is a repulsion effect? 
One important characteristic is the change in choice behavior as a function of decoy placement relative to the target in the two-dimensional attribute space. 6 We found that repulsion effects were strongest when the target and the decoy were most similar to each other. Larger distances in the attribute space between the target and the decoy moved behavior closer to the "attraction" end of the attraction-repulsion continuum, a pattern that was pronounced both in the perceptual and the preferential condition. Other studies in perceptual (Spektor et al., 2018) and preferential decision making (Dumbalska et al., 2020, Soltani et al., 2012) reported similar observations (but see Izakson et al., 2020). Notably, Liao et al. (2021) found a "wavy" pattern, where the respective context effect (attraction effect in preferential and inference tasks [Exp. 2]; repulsion effect in perceptual tasks [Exp. 1]) weakens as the attribute distance increases, flips into the other effect, and then flips one more time to become the original effect yet again.

Compared to the present experiment, Liao et al.'s (2021) manipulations of the target-decoy attribute distance spanned a larger range, starting at $9 \%$ and going as high as 94\%. In their perceptual-choice condition (Exp. 1), Liao and colleagues reported consistent repulsion effects for all distances of $24 \%$ or lower, which align with the results from the present experiment. However, in their preferential-choice condition (a consumer choice task [Exp. 2b]), repulsion effects were only observed when the decoy was substantially inferior to the target (in the range of $64 \%$ and $84 \%$ difference). In stimulus-distance conditions comparable to our study, strong attraction effects occurred. It is difficult to see how these discrepant results can be reconciled, given that Liao and colleagues varied both task framing (perceptual vs. preferential) and presentation format, and relied on a between-subjects design. Nevertheless, the differences in presentation format are the most likely culprit: Numerical representations of stimuli are known to give rise to attraction effects, whereas non-numeric perceptual features inhibit them (Frederick et al., 2014, Huber et al., 2014; Spektor et al., 2021; Spektor et al., 2018).

\footnotetext{
${ }^{6}$ We report additional characteristics in Appendix section "Characterizing the repulsion effect,'
} 


\section{Conclusion}

For a long time, repulsion effects have been treated by researchers as highly implausible phenomena, to the extent that they would cast a shadow of doubt over any theoretical account that dared to include them as a possibility (Tsetsos et al., 2015). This skepticism overlooked the influence of presentation format on choice, which can reverse long-standing context effects even modest manipulations are applied (Cataldo \& Cohen, 2019; Spektor et al., 2018). As it stands, whether decoy options increase or decrease the choice share of the options that dominate them, depends to a large extent on how information is presented to the decision maker (Spektor et al., 2021). This dependency is not limited to perceptual choices - it also holds in the preferential-choice domain.

These results, together with the failure of the MLBA model to account for them, suggests that research on context effects has taken a somewhat myopic view on the phenomena at hand. Many researchers have tacitly assumed that experimental designs and task domains are mostly interchangeable, provided that stimulus attributes can be used to represent options as illustrated in Figure 1. In other words, consumer choices and perceptual choices can be treated in the exact same way by virtue of an analogical relationship. To be clear, there is nothing wrong with this approach per se - after all, analogical reasoning plays a critical role in scientific reasoning (Hesse, 1966). However, researchers are still required to investigate whether the specifics surrounding each choice (which are being left out by the analogy) are indeed negligible. In other words, they need to ensure that they have the "model of the experiment" (Kellen, 2019). Failure to do so can lead to situations in which researchers hold distorted views of phenomena and rely on past modeling 'successes' that are driven by idiosyncratic experimental-design features presumed to be unrelated with the substantive research question. Our investigations on the occurrence of repulsion effects shows is that idiosyncrasies of different choice tasks cannot be ignored (see Spektor et al., 2018). What this means is that, despite the tremendous efforts towards the observation and characterization of context effects in recent years, our current level of understanding is still quite 
unsatisfactory — research programs should adjust accordingly. 


\section{Acknowledgments}

The authors acknowledge financial support from the German Research Foundation (DFG) through the Reinhart Koselleck Project (grant DFG Kl 614/39-1 to Karl Christoph Klauer). 


\section{References}

Annis, J., Miller, B. J., \& Palmeri, T. J. (2017). Bayesian inference with Stan: A tutorial on adding custom distributions. Behavior Research Methods, 49(3), 863-886. https://doi.org/10.3758/s13428-016-0746-9

Berkowitsch, N. A. J., Scheibehenne, B., \& Rieskamp, J. (2014). Rigorously testing multialternative decision field theory against random utility models. Journal of Experimental Psychology: General, 143(3), 1331-1348. https://doi.org/10.1037/a0035159

Brown, S. D., \& Heathcote, A. (2008). The simplest complete model of choice response time: Linear ballistic accumulation. Cognitive Psychology, 57(3), 153-178. https://doi.org/10.1016/j.cogpsych.2007.12.002

Busemeyer, J. R., Gluth, S., Rieskamp, J., \& Turner, B. M. (2019). Cognitive and neural bases of multi-attribute, multi-alternative, value-based decisions. Trends in Cognitive Sciences, 23(3), 251-263. https://doi.org/10.1016/j.tics.2018.12.003

Carpenter, B., Gelman, A., Hoffman, M. D., Lee, D., Goodrich, B., Betancourt, M., Brubaker, M., Guo, J., Li, P., \& Riddell, A. (2017). Stan: A probabilistic programming language. Journal of Statistical Software, $76(1), 1-32$. https://doi.org/10.18637/jss.v076.i01

Castillo, G. (2020). The attraction effect and its explanations. Games and Economic Behavior, 119, 123-147. https://doi.org/10.1016/j.geb.2019.10.012

Cataldo, A. M., \& Cohen, A. L. (2019). The comparison process as an account of variation in the attraction, compromise, and similarity effects. Psychonomic Bulletin \& Review, 26(3), 934-942. https://doi.org/10.3758/s13423-018-1531-9

Cataldo, A. M., \& Cohen, A. L. (2020). Modeling preference reversals in context effects over time. Computational Brain \& Behavior. https://doi.org/10.1007/s42113-020-00078-8

Cohen, A. L., Kang, N., \& Leise, T. L. (2017). Multi-attribute, multi-alternative models of choice: Choice, reaction time, and process tracing. Cognitive Psychology, 98, 45-72. https://doi.org/10.1016/j.cogpsych.2017.08.001 
Dumbalska, T., Li, V., Tsetsos, K., \& Summerfield, C. (2020). A map of decoy influence in human multialternative choice. Proceedings of the National Academy of Sciences, 117 (40), 25169-25178. https://doi.org/10.1073/pnas.2005058117

Dutilh, G., \& Rieskamp, J. (2016). Comparing perceptual and preferential decision making. Psychonomic Bulletin \&5 Review, $23(3)$, 723-737. https://doi.org/10.3758/s13423-015-0941-1

Evangelidis, I., Levav, J., \& Simonson, I. (2018). The asymmetric impact of context on advantaged versus disadvantaged options. Journal of Marketing Research, 55(2), 239-253. https://doi.org/10.1509/jmr.14.0483

Evans, N. J., Holmes, W. R., Dasari, A., \& Trueblood, J. S. (2021). The impact of presentation order on attraction and repulsion effects in decision-making. Decision, 8(1), 36-54. https://doi.org/10.1037/dec0000144

Evans, N. J., Holmes, W. R., \& Trueblood, J. S. (2019). Response-time data provide critical constraints on dynamic models of multi-alternative, multi-attribute choice. Psychonomic Bulletin \& Review, 26(3), 901-933. https://doi.org/10.3758/s13423-018-1557-z

Farmer, G. D., Warren, P. A., El-Deredy, W., \& Howes, A. (2017). The effect of expected value on attraction effect preference reversals. Journal of Behavioral Decision Making, 30(4), 785-793. https://doi.org/10.1002/bdm.2001

Frederick, S., Lee, L., \& Baskin, E. (2014). The limits of attraction. Journal of Marketing Research, 51(4), 487-507. https://doi.org/10.1509/jmr.12.0061

Gluth, S., Hotaling, J. M., \& Rieskamp, J. (2017). The attraction effect modulates reward prediction errors and intertemporal choices. Journal of Neuroscience, 37(2), 371-382. https://doi.org/10.1523/JNEUROSCI.2532-16.2017

Gluth, S., Spektor, M. S., \& Rieskamp, J. (2018). Value-based attentional capture affects multi-alternative decision making. eLife, $7,1-36$. https://doi.org/10.7554/eLife.39659

Hancock, T. O., Hess, S., Marley, A., \& Choudhury, C. F. (2021). An accumulation of preference: Two alternative dynamic models for understanding transport choices. 
Transportation Research Part B: Methodological, 149, 250-282. https://doi.org/10.1016/j.trb.2021.04.001

Herne, K. (1999). The effects of decoy gambles on individual choice. Experimental Economics, 2, 31-40. https://doi.org/10.1023/A:1009925731240

Hesse, M. B. (1966). Models and Analogies in Science. Notre Dame University Press.

Huber, J., Payne, J. W., \& Puto, C. P. (1982). Adding asymmetrically dominated alternatives: Violations of regularity and the similarity hypothesis. Journal of Consumer Research, 9(1), 90-98. https://doi.org/10.1086/208899

Huber, J., Payne, J. W., \& Puto, C. P. (2014). Let's be honest about the attraction effect. Journal of Marketing Research, 51(4), 520-525. https://doi.org/10.1509/jmr.14.0208

Izakson, L., Zeevi, Y., \& Levy, D. J. (2020). Attraction to similar options: The Gestalt law of proximity is related to the attraction effect (T. Davis, Ed.). PLOS ONE, 15(10), 1-21. https://doi.org/10.1371/journal.pone.0240937

Kellen, D. (2019). A model hierarchy for psychological science. Computational Brain 8 Behavior, 2(3-4), 160-165.

Kellen, D., Steiner, M. D., Davis-Stober, C. P., \& Pappas, N. R. (2020). Modeling choice paradoxes under risk: From prospect theories to sampling-based accounts. Cognitive Psychology, 118, 101258.

Kreps, D. M. (1990). A course in microeconomic theory. Princeton University Press.

Latty, T., \& Beekman, M. (2011). Irrational decision-making in an amoeboid organism: transitivity and context-dependent preferences. Proceedings of the Royal Society B: Biological Sciences, 278(1703), 307-312.

https://doi.org/10.1098/rspb.2010.1045

Liao, J., Chen, Y., Lin, W., \& Mo, L. (2021). The influence of distance between decoy and target on context effect: Attraction or repulsion? Journal of Behavioral Decision Making, 34(3), 432-447. https://doi.org/10.1002/bdm.2220 
Lichters, M., Bengart, P., Sarstedt, M., \& Vogt, B. (2017). What really matters in attraction effect research: when choices have economic consequences. Marketing Letters, 28(1), 127-138. https://doi.org/10.1007/s11002-015-9394-6

Liew, S. X., Howe, P. D. L., \& Little, D. R. (2016). The appropriacy of averaging in the study of context effects. Psychonomic Bulletin \& Review, 23(5), 1639-1646. https://doi.org/10.3758/s13423-016-1032-7

Molloy, M. F., Galdo, M., Bahg, G., Liu, Q., \& Turner, B. M. (2019). What's in a response time? On the importance of response time measures in constraining models of context effects. Decision, 6(2), 171-200. https://doi.org/10.1037/dec0000097

Noguchi, T., \& Stewart, N. (2014). In the attraction, compromise, and similarity effects, alternatives are repeatedly compared in pairs on single dimensions. Cognition, 132(1), 44-56. https://doi.org/10.1016/j.cognition.2014.03.006

Noguchi, T., \& Stewart, N. (2018). Multialternative decision by sampling: A model of decision making constrained by process data. Psychological Review, 125(4), 512-544. https://doi.org/10.1037/rev0000102

Oppenheimer, D. M., \& Kelso, E. (2015). Information processing as a paradigm for decision making. Annual review of psychology, 66, 277-294.

Parrish, A. E., Evans, T. A., \& Beran, M. J. (2015). Rhesus macaques (Macaca mulatta) exhibit the decoy effect in a perceptual discrimination task. Attention, Perception, ES Psychophysics, $77(5), 1715-1725$. https://doi.org/10.3758/s13414-015-0885-6

Regenwetter, M., \& Robinson, M. M. (2017). The construct-behavior gap in behavioral decision research: A challenge beyond replicability. Psychological Review, 124(5), 533-550. https://doi.org/10.1037/rev0000067

Shimojo, S., Simion, C., Shimojo, E., \& Scheier, C. (2003). Gaze bias both reflects and influences preference. Nature Neuroscience, 6(12), 1317-1322. https://doi.org/10.1038/nn1150 
Simonson, I. (2014). Vices and virtues of misguided replications: The case of asymmetric dominance. Journal of Marketing Research, 51 (4), 514-519. https://doi.org/10.1509/jmr.14.0093

Singmann, H., \& Kellen, D. (2013). MPTinR: Analysis of multinomial processing tree models in R. Behavior Research Methods, 45(2), 560-575. https://doi.org/10.3758/s13428-012-0259-0

Smith, S. M., \& Krajbich, I. (2018). Attention and choice across domains. Journal of Experimental Psychology: General, 147(12), 1810-1826. https://doi.org/10.1037/xge0000482

Soltani, A., De Martino, B., \& Camerer, C. (2012). A range-normalization model of context-dependent choice: A new model and evidence. PLOS Computational Biology, 8(7), 1-15. https://doi.org/10.1371/journal.pcbi.1002607

Spektor, M. S., Bhatia, S., \& Gluth, S. (2021). The elusiveness of context effects in decision making. Trends in Cognitive Sciences, 25(10), 844-857. https://doi.org/10.1016/j.tics.2021.07.011

Spektor, M. S., Kellen, D., \& Hotaling, J. M. (2018). When the good looks bad: An experimental exploration of the repulsion effect. Psychological Science, 29(8), 1309-1320. https://doi.org/10.1177/0956797618779041

Spitmaan, M., Chu, E., \& Soltani, A. (2019). Salience-driven value construction for adaptive choice under risk. The Journal of Neuroscience, 39(26), 5195-5209. https://doi.org/10.1523/JNEUROSCI.2522-18.2019

Tom, S. M., Fox, C. R., Trepel, C., \& Poldrack, R. A. (2007). The neural basis of loss aversion in decision-making under risk. Science, 315(5811), 515-518. https://doi.org/10.1126/science.1134239

Trueblood, J. S., Brown, S. D., \& Heathcote, A. (2014). The multiattribute linear ballistic accumulator model of context effects in multialternative choice. Psychological Review, 121(2), 179-205. https://doi.org/10.1037/a0036137 
Trueblood, J. S., Brown, S. D., \& Heathcote, A. (2015). The fragile nature of contextual preference reversals: Reply to Tsetsos, Chater, and Usher (2015). Psychological Review, 122(4), 848-853. https://doi.org/10.1037/a0039656

Trueblood, J. S., Brown, S. D., Heathcote, A., \& Busemeyer, J. R. (2013). Not just for consumers: Context effects are fundamental to decision making. Psychological Science, 24(6), 901-908. https://doi.org/10.1177/0956797612464241

Trueblood, J. S., \& Pettibone, J. C. (2017). The phantom decoy effect in perceptual decision making. Journal of Behavioral Decision Making, 30(2), 157-167. https://doi.org/10.1002/bdm.1930

Tsetsos, K., Chater, N., \& Usher, M. (2012). Salience driven value integration explains decision biases and preference reversal. Proceedings of the National Academy of Sciences of the United States of America, 109(24), 9659-9664. https://doi.org/10.1073/pnas.1119569109

Tsetsos, K., Chater, N., \& Usher, M. (2015). Examining the mechanisms underlying contextual preference reversal: Comment on Trueblood, Brown, and Heathcote (2014). Psychological Review, 122(4), 838-847. https://doi.org/10.1037/a0038953

Tsetsos, K., Moran, R., Moreland, J., Chater, N., Usher, M., \& Summerfield, C. (2016). Economic irrationality is optimal during noisy decision making. Proceedings of the National Academy of Sciences of the United States of America, 113(11), 3102-3107. https://doi.org/10.1073/pnas.1519157113

Turner, B. M., Schley, D. R., Muller, C., \& Tsetsos, K. (2018). Competing theories of multialternative, multiattribute preferential choice. Psychological Review, 125(3), 329-362. https://doi.org/10.1037/rev0000089

Tversky, A. (1972). Elimination by aspects: A theory of choice. Psychological Review, 79 (4), 281-299. https://doi.org/10.1037/h0032955

Vanunu, Y., Hotaling, J. M., \& Newell, B. R. (2020). Elucidating the differential impact of extreme-outcomes in perceptual and preferential choice. Cognitive Psychology, 119, 101274. https://doi.org/10.1016/j.cogpsych.2020.101274 
Walasek, L., \& Stewart, N. (2015). How to make loss aversion disappear and reverse: Tests of the decision by sampling origin of loss aversion. Journal of Experimental Psychology: General, 144(1), 7-11. https://doi.org/10.1037/xge0000039

Wu, S., \& Yu, R. (2020). The impact of phantom decoys on the neural processing of valuation. Brain Structure and Function, (Trueblood 2012). https://doi.org/10.1007/s00429-020-02079-6

Yang, S., \& Lynn, M. (2014). More evidence challenging the robustness and usefulness of the attraction effect. Journal of Marketing Research, 51(4), 508-513. https://doi.org/10.1509/jmr.14.0020

Zeigenfuse, M. D., Pleskac, T. J., \& Liu, T. (2014). Rapid decisions from experience. Cognition, 131(2), 181-194. https://doi.org/10.1016/j.cognition.2013.12.012 


\section{Appendix}

\section{Robustness checks}

\section{Transfer effects}

In the present study, we controlled for any potential influence of perceptual features by representing lotteries in the preferential condition using the same minimalist stimuli as in the perceptual condition. Even though such a simplified representation is quite common in the risky-choice literature (e.g., Farmer et al., 2017; Gluth et al., 2018, Spitmaan et al., 2019, Tsetsos et al., 2016), it is possible that individuals who encountered the perceptual condition first ignored the instructions and treated the preferential condition as if it was perceptual. If this was the case, the similarity in behavior across both conditions would not reflect the similarity between preferential and perceptual choices but rather a trivial repetition of the same task.

To test this possibility, we repeated the context-effect analysis using the data from the first session only. In the perceptual condition, the median of the RST was .478 (95\% BCI: $[.470, .485])$ and in the preferential condition, the median was $.487(95 \%$

BCI: $[.473, .502])$. Note that these analyzes are noisier as they are based only on half of the sample. That said, the results remained both qualitatively and quantitatively largely the same, even though the $95 \%$ BCI in the preferential condition now overlaps with .50 . With respect to the main experimental manipulations, choice behavior is strikingly similar between the both sessions and the first session only (Figure A1).

\section{Task engagement}

The present study comprised of 700 trials per experimental session, amounting to an average of a little more than an hour per session (including three self-paced breaks) and a substantial amount of inter-individual variability. Even though both the trial count and the duration are normal for experimental studies, participants' movements were somewhat restricted due to the eye recordings, making it potentially more tiring than purely behavioral experiments. We evaluated different possibilities of how this might have affected behavior. 
(a) Perceptual condition
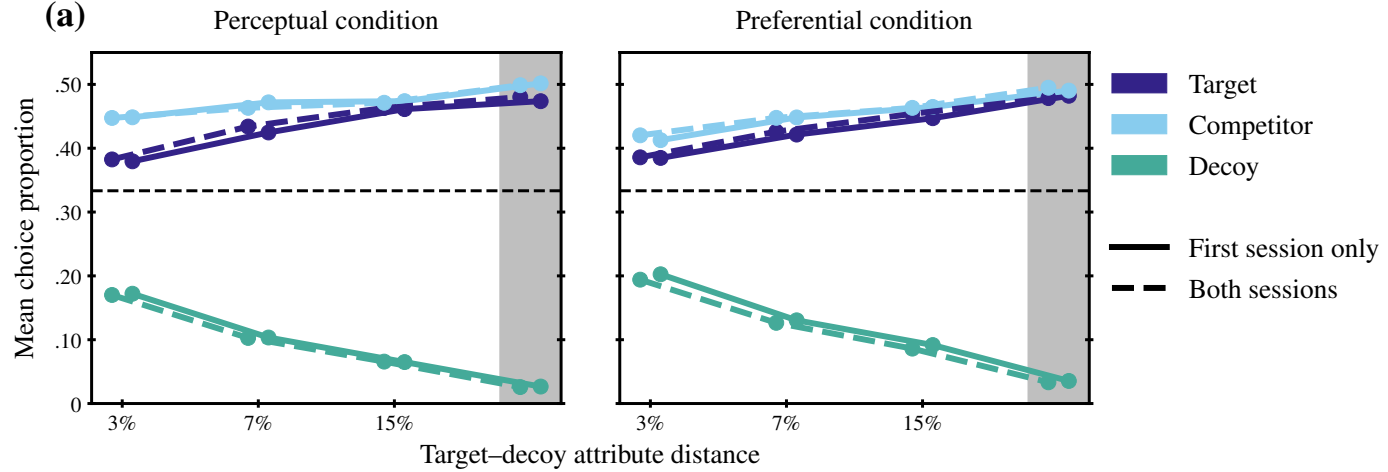

(b)
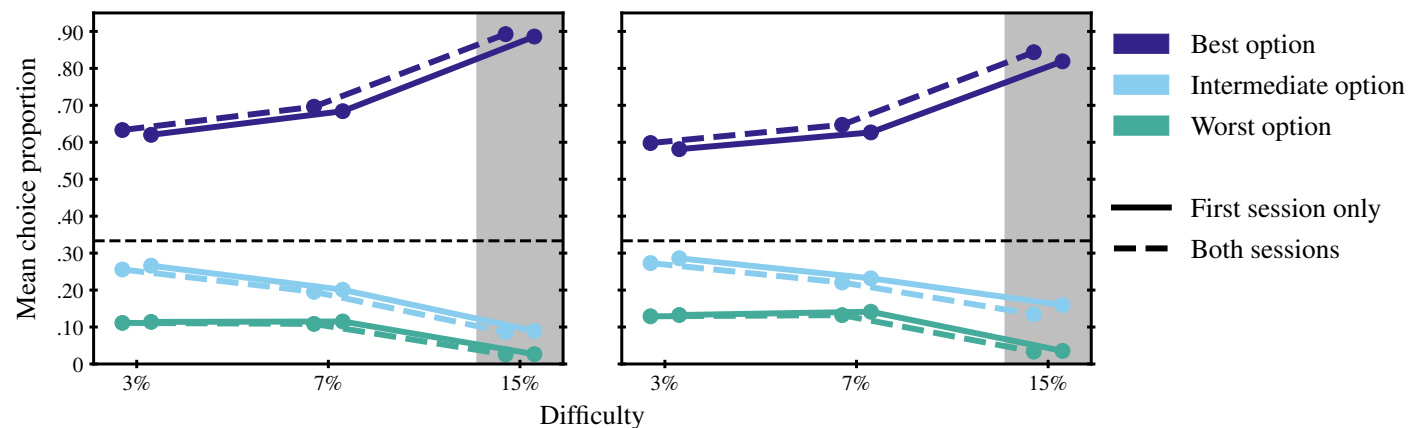

\section{Figure A1}

Mean choice proportions of the options, split by condition and (a) target-decoy attribute distance and (b) choice difficulty. The dashed lines reproduce the mean choice proportions from Figure 4. The solid lines represent choice proportions in the first session only. Lines are horizontally jittered and the error bars omitted for clarity.

As a first possibility, variation in task-completion times might be systematically related to variation in behavior. For example, participants who took a long time to complete the experiments were the ones that had to re-calibrate the eye tracker more frequently, took longer breaks due to fatigue, and so on, potentially becoming frustrated and shifting their strategy to a heuristic one to finish the experiment quicker. In such a case, participants who completed the experiment quicker should be "better" than those whose motivation was high in the beginning and declined as the experiment progressed. Two observations suggest that this possibility is rather unlikely. First, we find little evidence that the experiment-completion time was driven by factors other than average response times, as the two measures were highly correlated $(r=.77)$. Second, we found sizeable positive correlations between experiment-completion times and accuracy (i.e., 

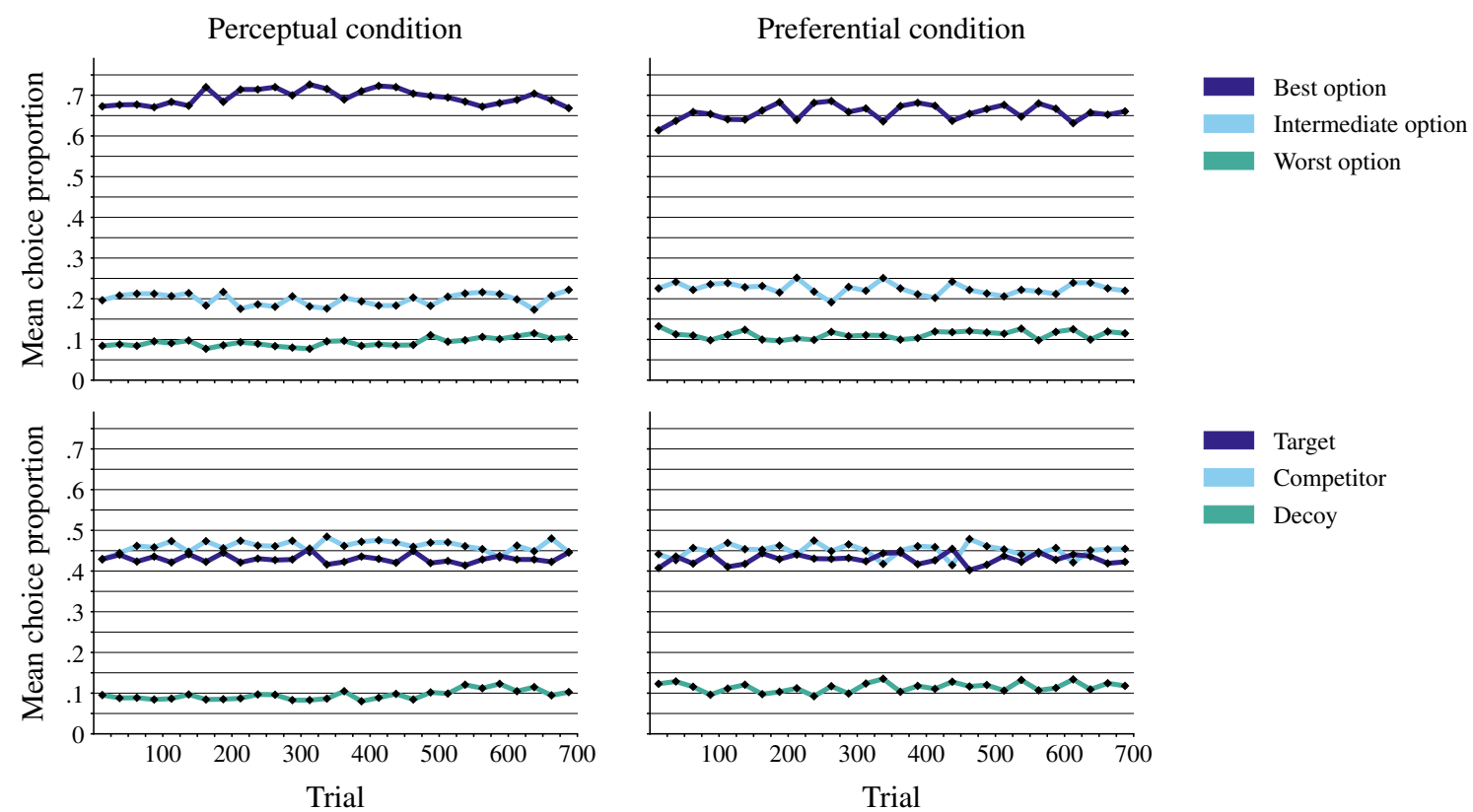

\section{Figure A2}

Temporal development of choice proportions in bins of 25 trials. The top row shows choice proportions according to accuracy and the bottom row shows choice proportions according to the function of each option.

proportion of choosing the option with the highest criterion or expected value), both split by condition (perceptual condition: $r=.40$, preferential condition: $r=.60$ ) and split by session (Session 1: $r=.60$, Session 2: $r=.62$ ). Both observations suggest that the main determinant of experiment-completion time was the speed-accuracy trade-off adopted by the individuals.

As a second possible effect, individuals might have become tired as the experiment progressed, trading off accuracy in favor of faster decisions, becoming less accurate in general, or adopting some heuristic choice strategies. To evaluate such a possibility, we have split the experiment into bins of 25 trials and looked for temporal dependencies in terms of accuracy (Figure A2, top row) and tendency to choose specific options (Figure A2, bottom row). Visual inspection of these temporal developments reveals that there is only a negligible influence of trial number on choices. Taken together, we found that individuals seem to have taken the task seriously both within and across the two experimental sessions, without evidence of fatigue or strategy shifts. 


\section{Characterizing the repulsion effect}

Even though existing models of multi-alternative choice, such as the multiattribute linear ballistic accumulator model (MLBA), predict repulsion effects in some specific situations (Tsetsos et al., 2015), so far, there exists no dedicated formal account of the repulsion effect. To guide future theory development, we report additional analyses that provide a more in-depth characterization of the behavior underlying repulsion effects.

\section{Similarity or repulsion?}

The similarity effect (Tversky, 1972), according to which similar options take away choice shares from each other, predicts behavior that is phenomenologically indistinguishable from a repulsion effect, as both predict an increased choice proportion of dissimilar options. However, the handful of papers that postulate a mechanism of the repulsion effect (Frederick et al., 2014, p. 493; Kreps, 1990, p. 28; Simonson, 2014, p. 518) agrees that the detection of the dominance relationship between the target and the decoy is essential for repulsion effects to occur. Therefore, even though both similarity and repulsion effects reduce the choice share of the more similar core option relative to the dissimilar one, there are important differences in their corollary effects: Whereas the similarity effect leads to the treatment of the similar core option and the decoy as rough substitutes of each other, the repulsion effect establishes a clear dominance relationship between them. Here, we provide additional analyses that evaluate the possibility of similarity effects. Foreshadowing the results, we find little evidence that people fail to detect the dominance relationship between the target and the decoy.

As a first analysis, we looked at the condition in which similarity effects are most likely to arise, namely, when the target and the decoy are most similar to each other ( $2 \%$ attribute distance; see Figure 4 ). Even in such a situation, participants chose the target over the decoy. Similarly, in the most difficult condition in which the two core options differed by $3 \%$ (Figure $4 \mathrm{~b})$, the best option was chosen more than twice as often 
as the second-best option. On the other hand, among all trials used in the present experiment, the ones in which the attribute distance between the target and the decoy was 20\% (124 trials that were used as "catch trials") is the least susceptible to the similarity effect: On these trials, the decoy was chosen on average in only $2.4 \%$ of the cases $(S D=1.8 \%)$. Nevertheless, using catch trials only, people still chose the target less often than the competitor (95\% BCI in the perceptual condition: [.483, .498]; 95\% BCI in the preferential condition: $[.482, .500])$. This is particularly remarkable given that this analysis is based on less than $18 \%$ of the available data and with an average choice rate of the best option as high as $87 \%$.

In the following analysis, we treated the trials as the focal observational unit: Across the 700 unique trials (with 104-110 observations each, coming from the 55 participants and both conditions), is there any evidence for individuals systematically mixing up the target and the decoy? Across all 700 trials, the target has been chosen significantly more often than the decoy on 568 (81.1\%; binomial test with a significance threshold of $5 \%$ ). None of the 7 trials on which the decoy was chosen more often than the target were significant (all $p \mathrm{~s} \geq .63$ ). Conditional on trials with sufficient statistical power (i.e., trials on which either the target or the decoy has been chosen at least half of the time), the target has been chosen significantly more often than the decoy in $94.4 \%$ of the trials.

Finally, one could argue that the apparent repulsion effect could result from a mixture of other effects such that people show similarity effects on some trials, attraction effects on some other trials, and so on. We cannot exclude this possibility given that mixture accounts can be extremely flexible and therefore nearly impossible to reject under most experimental designs. As a remedy, one can set up an identifiable mixture account and evaluate the parameter estimates obtained (see Singmann \& Kellen, 2013). Taking this approach, we found that the repulsion effects illustrated in Figure 4 can be mimicked by a mixture of two processes: (1) when choosing between the three options, the target and decoy are perceived as equivalent and are chosen with equal probabilities (i.e., there is a similarity effect), and (2) the dominance relationship 


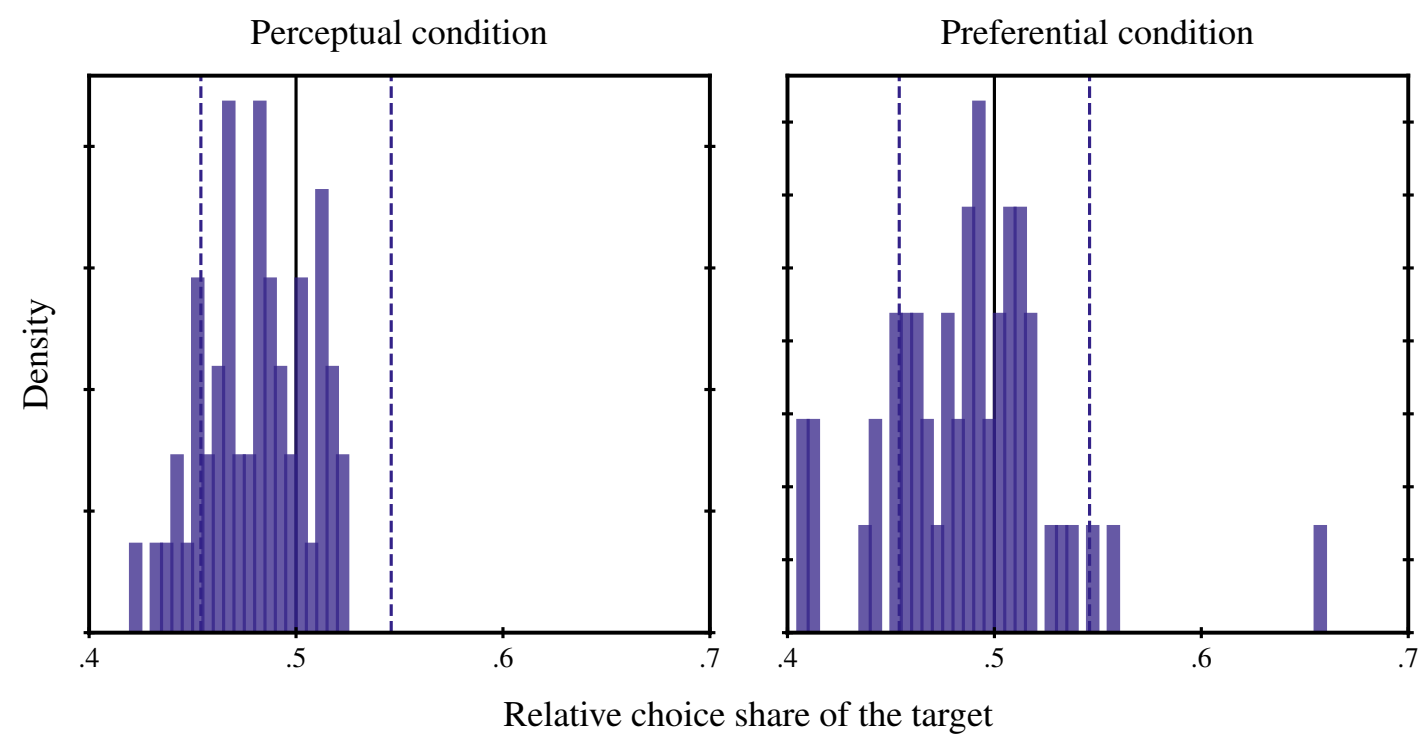

\section{Figure A3}

Distribution of relative choice shares of the target, split by condition. The dashed lines represent the thresholds for the lowest/highest values that were significant at the individual level.

between the target and the decoy is detected such that the latter is excluded from consideration (there is a choice between the target and the competitor only). Under this mixture characterization, the probability estimate for scenario (1) taking place in the most difficult perceptual/preferential condition (2\% difficulty, $3 \%$ target-decoy attribute distance) is only $.52 / .55$, respectively. These estimates are considerably low given that this is the condition in which scenario (1) is expected to occur most often. In contrast, the analogous estimated probability in the catch trials (15\% difficulty, $20 \%$ target-decoy attribute distance) is $.08 / .10$, respectively. These mixture probability estimates strike us as implausible: They are considerably low when the task is exceedingly difficult and somewhat high when the task is trivial (and the decoy is, in fact, almost never chosen).

\section{Central tendency or individual differences?}

Related to the previous paragraph, a mixture model that assumes dominance detection on some trials and similarity effects on others stands at odds with existing research that consistently found these two forces to inhibit each other (Berkowitsch et al., 2014, Liew et al., 2016, Trueblood et al., 2015; Turner et al., 2018). While this is 
true for analyses within individuals, a mixture of attraction and repulsion effects across individuals is theoretically a lot more plausible. Do we find evidence for such a mixture of effects? If this was the case, the interpretation of the results presented in the manuscript would be subject to a classical aggregation fallacy (Regenwetter \& Robinson, 2017), according to which aggregate behavior is not representative of the behavior of any individual.

We used a binomial test to assess, for each individual and condition separately, whether the proportion of target choices (relative to choices of the target or the competitor, i.e., without too-slow responses or decoy choices) significantly differed from $50 \%$. Of the 23 significant binomial tests, 20 were individuals who showed a repulsion effect and only 3 who showed attraction effects. A visual inspection of the distribution of the relative choice shares of the target (Figure A3) reveals unimodality and a shift to the left of the $50 \%$ mark. In sum, we find little evidence for an artefactual occurrence of a repulsion effect on the aggregate level. Instead, it seems to be a consistent tendency to select the dissimilar option, despite a high level of dominance detection.

\section{Alternative specifications of the multiattribute linear ballistic accumulator model}

In many situations, the MLBA has been shown to provide a good fit to choices and the associated latencies (Cataldo \& Cohen, 2020; Evans et al., 2019; but see Cohen et al., 2017; Turner et al., 2018, for cases where it did not perform that well). In the present study, its ability to account for the observed behavior fell somewhat short of the expectations. Here, we explore two potential sources of this sub-par performance by considering a model that has a separate set of parameters for different cells of the experimental design and a model that does not have to explain response times on top of choices.

\section{A maximally flexible version of the model}

In order to determine whether the MLBA is in principle unable to account for the behavioral patterns observed or whether its specification is too restrictive, we 
(a)

Perceptual condition
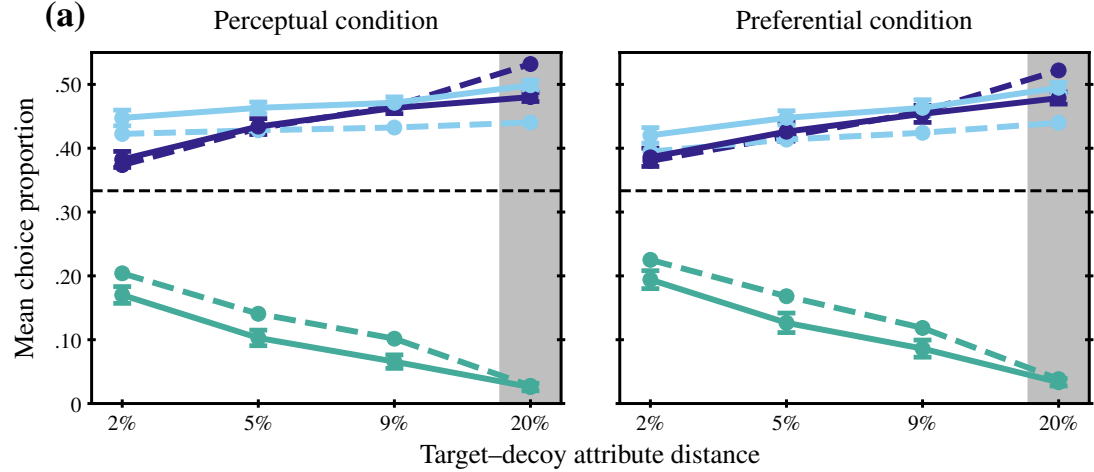

Target
Competitor

Decoy

Data

- MLBA predictions

(b)
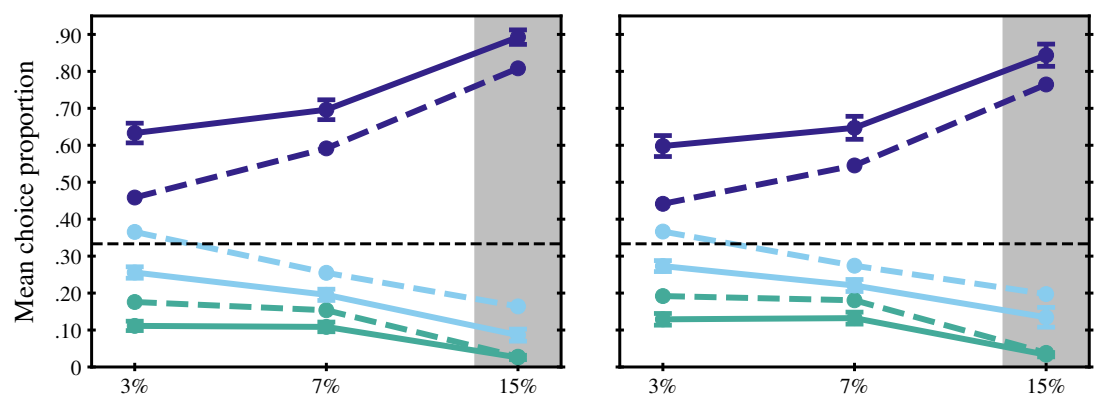

Best option

Intermediate option

Worst option

Data

- MLBA predictions

Difficulty

\section{Figure A4}

Mean choice proportions of the options and corresponding model predictions, split by condition and (a) target-decoy attribute distance and (b) choice difficulty. Target is the option that is similar to the decoy option but dominating it, whereas competitor is the option that is dissimilar to both target and decoy. All distance measures are in percentages of the maximum criterion value of 400. The conditions that were used as catch trials are highlighted by a shaded background. Model predictions reflect mean posterior predictions of the multiattribute linear ballistic accumulator model that was fit to separate cells of the experimental design. See Appendix section A maximally flexible version of the model" for details.

assessed a maximally flexible version of the model by estimating separate sets of parameters for each of the six combinations of the experimental design factors target-decoy attribute distance (3 levels) and choice difficulty (2 levels), with 96 trials per person and session within each of them. Additionally, and in contrast to the other fitting procedures reported here, we also estimated the model using the data of the catch trials (124 trials), for a total of 63 parameters that are estimated for each person 


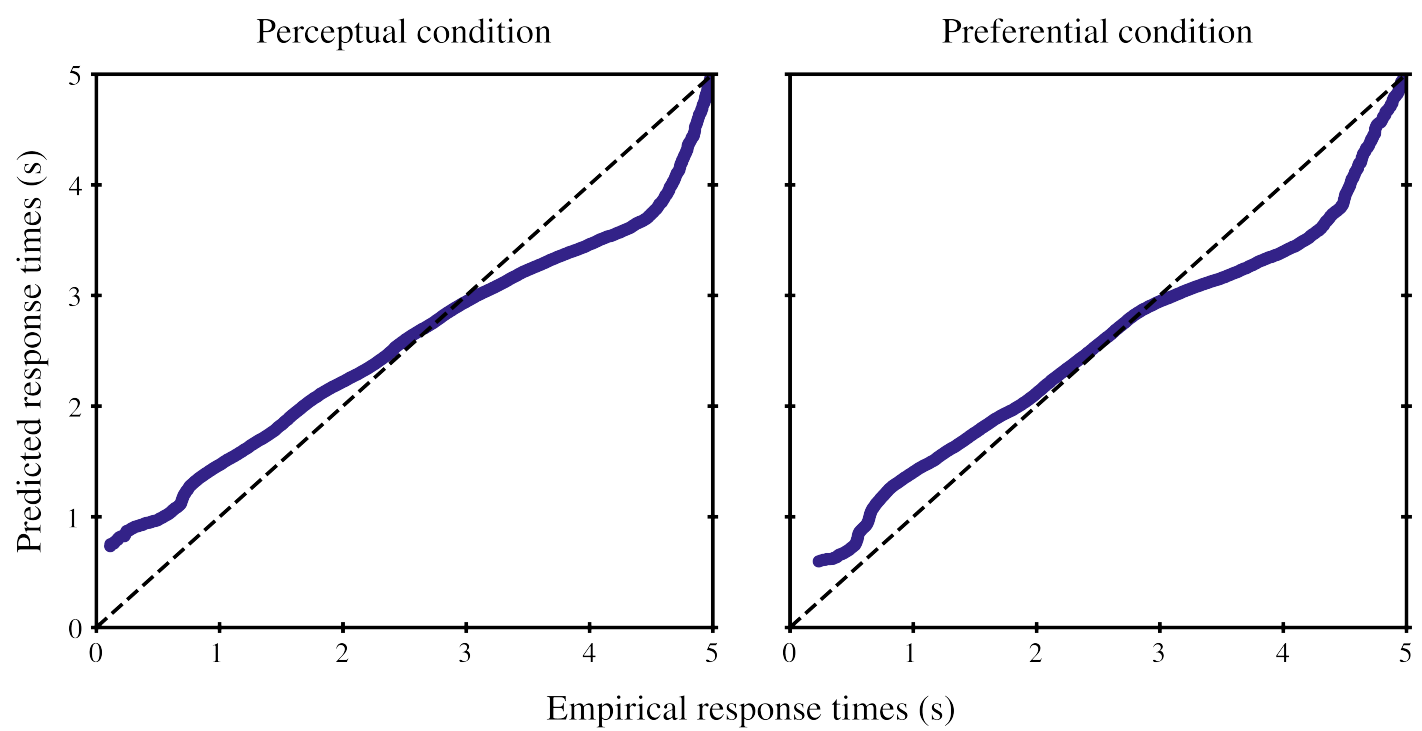

\section{Figure A5}

Quantile-quantile plots of empirical and predicted response times. Predictions are based on the mean of the posterior response-time predictions of the multiattribute linear ballistic accumulator model that was fit to separate cells of the experimental design. See Appendix section "A maximally flexible version of the model" for details.

and session. Despite the large number of free parameters and the flexibility inherent to it, the model still struggles to accommodate some behavioral patterns. Firstly, while it can (quantitatively) accommodate choice proportions on a functional level (i.e., whether an option is a target, competitor, or decoy; Figure A4 a) rather well, it struggles with predictions on a criterion level (i.e., whether an option has the highest criterion value; Figure A4p) and substantially under-predicts proportions of correct choices (e.g., 46\% vs. the observed $63 \%$ for the $3 \%$ difficulty level in the perceptual condition). Secondly, apart from the condition in which the target and the decoy differ by $2 \%$, it cannot accommodate repulsion effects in any of the other conditions, casting into doubt its ability to jointly explain repulsion effects and the latencies accompanying them. Finally, despite quantitative improvements in the model's ability to predict response times, the quantile-quantile plots of empirical and predicted response times (Figure A5 still show a pronounced inverse-S shape. 
(a)

Perceptual condition

Preferential condition

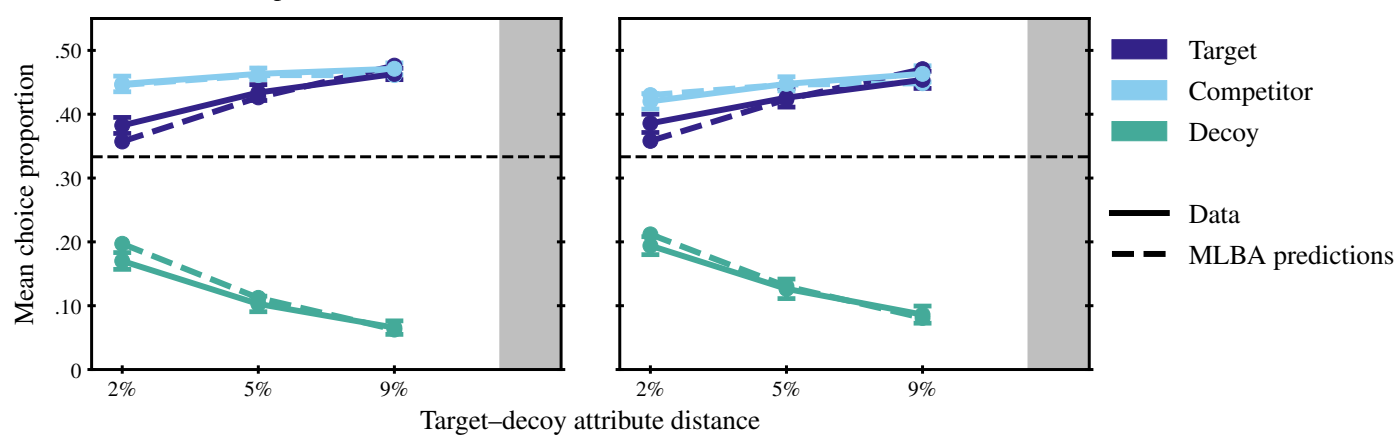

(b)
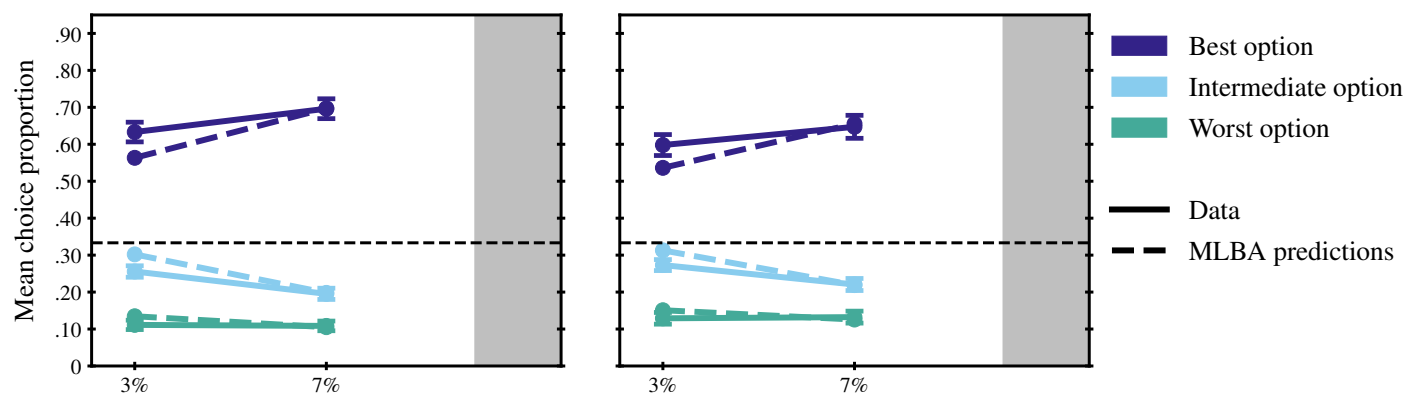

Difficulty

\section{Figure A6}

Mean choice proportions of the options and corresponding model predictions, split by condition and (a) target-decoy attribute distance and (b) choice difficulty. Target is the option that is similar to the decoy option but dominating it, whereas competitor is the option that is dissimilar to both target and decoy. All distance measures are in percentages of the maximum criterion value of 400. The conditions that were used as catch trials are highlighted by a shaded background and were excluded from model fitting. Model predictions reflect mean posterior predictions of the multiattribute linear ballistic accumulator model that was fit using a softmax choice function as the error model. See Appendix section A model without response times" for details.

\section{$A$ model without response times}

Decision-making models that are able to account for context effects have traditionally relied on an evidence-accumulation framework that predicts choices in conjunction with response times (see Busemeyer et al., 2019, for a recent review), and response times, in turn, are crucial for distinguishing and constraining the various models (Evans et al., 2019; Molloy et al., 2019). Whereas other prominent models rely 
on a single decision-making process, the MLBA has separate front-end and back-end processes that make it possible to combine the front-end process with a different error model, which has successfully been applied in the past (Hancock et al., 2021). Intriguingly, the MLBA has been shown to be able to predict repulsion effects in the popular rectangle-size task (Spektor et al., 2018) but it was fit to choice data only. We assessed whether the response times restrict the model's ability to predict the qualitative patterns observed in the present experiment by replacing the linear ballistic accumulator back-end with a softmax choice rule that directly predicts the choice probability $\operatorname{Pr}(i)$ of option $i$ from their respective drift rates $d_{i}$ :

$$
\operatorname{Pr}(i)=\frac{e^{\theta d_{i}}}{\sum e^{\theta d_{j}}}
$$

The inverse-temperature parameter $\theta$ governs the degree of maximization in the model and effectively replaces the parameters $I_{0}, \gamma, k, A$, and $\tau$, reducing the total number of free parameters to five.

We fitted this reduced model without response times to all choices (excluding catch trials) of each individual and session separately. In line with our past findings (Spektor et al., 2018), if the MLBA is not constrained by the response times, it does a remarkable job of explaining the behavioral patterns (see Figure A6). Importantly, even though the model was not endowed with the possibility to adjust its parameters within the various cells of the experimental design (as in Appendix section "A maximally flexible version of the model'), it was able to account for repulsion effects in all but the largest level of target-decoy attribute distance (in which it predicted only a very weak attraction effect). 